\title{
Landscape of Epithelial Mesenchymal Plasticity as an emergent property of coordinated teams in regulatory networks
}

Kishore Hari ${ }^{1}$, Varun Ullanat ${ }^{2}$, Archana Balasubramanian ${ }^{3 \dagger}$, Aditi Gopalan ${ }^{\dagger \dagger}$, Mohit Kumar Jolly ${ }^{{ }^{*}}$

${ }^{1}$ Centre for BioSystems Science and Engineering, Indian Institute of Science, Bangalore 560012, India

2 Department of Biotechnology, RV College of Engineering, Bangalore 560059, India

${ }^{3}$ Department of Biotechnology, PES University, Bangalore 560085, India

*Corresponding author: mkjolly@iisc.ac.in

${ }^{\dagger}$ Equal contributions 


\begin{abstract}
Elucidating the principles of cellular decision-making is of fundamental importance. These decisions are often orchestrated by underlying regulatory networks. While we understand the dynamics of simple network motifs, how do large networks lead to a limited number of phenotypes, despite their complexity, remains largely elusive. Here, we investigate five different networks governing epithelial-mesenchymal plasticity and identified a latent design principles in their topology that limits their phenotypic repertoire - the presence of two "teams" of nodes engaging in a mutually inhibitory feedback loop, forming a toggle switch. These teams are specific to these networks and directly shape the phenotypic landscape and consequently the frequency and stability of terminal phenotypes vs. the intermediary ones. Our analysis reveals that network topology alone can contain information about phenotypic distributions it can lead to, thus obviating the need to simulate them. We unravel topological signatures that can drive canalization of cell-fates during diverse decision-making processes.
\end{abstract}

Keywords: Network topology, complexity, robustness, phenotypic heterogeneity, Epithelial Mesenchymal Plasticity, Cellular decision making 


\section{Introduction}

Understanding the principles of cellular decision making is a fundamental question in cellular and developmental biology, with implications in mapping and eventually controlling cellular reprogramming and disease progression (1, 2, 3). These decisions are often orchestrated through the emergent dynamics of complex regulatory networks operating at multiple levels, including signaling, protein-protein interaction and transcriptional activation/inhibition. Nonlinear interactions in various such networks can enable emergent dynamics such as multistability and hysteresis (cellular memory) to facilitate adaptation to various stresses (4, 5). A better understanding of the underlying dynamics can also accelerate the design of synthetic circuits to achieve specific objectives. Thus, elucidating how specific regulatory networks lead to different emergent dynamics is instrumental for understanding how cells decide among multiple possible fates/phenotypes to choose for, and how such transitions can be controlled for directing cellular reprogramming to achieve certain desired scenarios, such as "differentiation therapy" (6) for cancers and or reprogramming pancreatic cells to make insulin (7).

Questions related to transition of cells from one state/phenotype to another reversibly or irreversibly during cellular differentiation have been investigated for almost five decades (8). We now know that many cellular decisions are often mediated by "network motifs" such as a toggle switch - a mutually inhibitory feedback loop between the two "master regulators" of sibling cell-fates (9). Such loops often drive two diverging decision-making trajectories in Waddington's landscape representing different possible terminal states a cell can converge to. Thus, a "toggle switch" between two nodes $A$ and $B$ leads to two states - (high A, low B) and (low A, high B) - each representing a different phenotype. However, decision-making may often involve much larger regulatory networks which can often involve multiple feedback loops. For instance, the global regulatory network in E.coli has approximately 150 transcription factors (TFs). Similarly, networks driving epithelialmesenchymal plasticity (EMP) in cancer cells can have over 50 players (10). Despite their complexity, many of these networks robustly lead to only a limited number of phenotypes, a process termed as "canalization" (11). This observation raises the question whether these networks constitute topological signatures that are capable of constraining the corresponding possible phenotypic repertoire.

We recently investigated the dynamics of a complex regulatory network (33 nodes, 357 edges) that led to only four phenotypes in small cell lung cancer (SCLC) (12). We demonstrated that this network consisted of two "teams"/groups of nodes such that members in a team activated each 
other directly/indirectly but members across teams inhibited each other. This topological feature reduced this complex network effectively into a "toggle switch" between teams, thus leading to a small number of phenotypes. However, many questions remain unanswered: a) can the presence of "teams" be witnessed in other regulatory networks? If yes, do they constrain the phenotypic space in those networks too? b) do these "teams" also make these biological networks/phenotypes robust to various perturbations? and c) can the team strength be used to predict the frequency of different phenotypes, without performing any dynamical simulations?

Here, we investigate the dynamics and topological hallmarks of five networks of different sizes, all implicated to regulate EMP during development as well as cancer progression. First, by analyzing their network topology, we found that all these networks consist of "teams" of nodes; one of these "teams" consist of drivers/stabilizers of mesenchymal phenotype, while the other one has those for epithelial phenotype. This "team" structure was largely lost upon disrupting the network topology by shuffling/randomization of edges. Second, our discrete, parameter-independent and continuous, parameter-agnostic simulations show that these "teams" are integral to stabilizing epithelial and mesenchymal phenotypes, as shown via various stability metrics. Thus, the hybrid epithelial/mesenchymal phenotypes were less frequent and also less resilient to dynamical perturbations. Finally, we show that the strength of teams in a regulatory network directly shapes the emergent phenotypic landscape, thus offering a network topology based metric to identify phenotypic distributions without performing any simulations. The topological signatures and metrics identified here can be applied to other instances of cellular decision-making as well to unravel their underlying fundamental dynamic hallmarks.

\section{Results}

\subsection{EMP networks display two teams of nodes consisting of EMT and MET inducers separately}

We started with a collection of networks of various sizes ranging from 18 nodes 33 edges (18N $33 E$ ) to 57 nodes 113 edges (57N 113E), reported to be involved in EMP (Fig 1A, S1). All these networks have been shown to exhibit epithelial and mesenchymal phenotypes that were largely exclusive in terms of which genes/nodes were "ON" (relatively higher levels) and "OFF" (relatively lower levels) (13, 14, 15, 16, 10). Given the size and complexity of these networks, it was surprising to see such a limited number of phenotypes with mutually exclusive expression patterns. Our recent work in small cell lung cancer (SCLC) showed that a highly complex network can have a limited number of phenotypes when the nodes in the network form a "team" and consequently the 
network can be reduced or coarse-grained to a small one. In such a structure, largely speaking, members of a "team" activate each other but members across teams inhibit mutually. Thus, the network can be seen similar to a "toggle switch" between two teams of players.

We hypothesized that these EMP networks consist of similar "teams" of nodes and that these nodes underlie their limited phenotypic repertoire. Unlike the SCLC network, these EMP networks were highly sparse, i.e. only a few nodes in a network directly regulated one another (Fig 1B, left). Thus, we decided to use the pairwise influence among the nodes of these networks (Fig 1B, right) rather than just the direct interactions among them, to analyse the structure of these networks. This "influence matrix" represents the effective regulation of one node by another when many different indirect paths are also counted (up to path length < 10) in addition to a direct regulation. The longer a path is, the less its weightage in terms of calculating the influence of a path from one node to another.

In the influence matrix, the nodes of these networks can be classified into 2 categories: peripheral nodes which include input and output nodes (grey boxes in Fig 1B(right)), and core nodes (red and blue boxes in Fig 1B(right)). Input nodes are defined as the nodes that are not influenced by any other nodes in the network (miR-205, miR-9, miR-30c in 22N82E network). Similarly, output nodes are defined as the nodes that do not influence any other nodes in the network (CDH1, VIM, KLF8, TCF3 in 22N82E network). On the contrary, core nodes in a network are both influenced by and influence other nodes in a network. The core nodes of all five EMP networks could further be classified into two groups of nodes using hierarchical clustering of the influence matrix (see Methods) (Fig 1B(right), S2A). Nodes belonging to the same group have a positive influence on each other (i.e. mutually activatory), while those belonging to different groups have a negative influence on each other (i.e. mutually inhibitory). In 22N82E network, these two groups or "teams" constitute of all EMT inducers in one team (TWIST1/2, GSC, FOXC2, ZEB1/2, SNAI1/2, TGFb) and all MET inducers in another (miR-200a/b/c, miR-34a, miR-141, miR-101) (17, 18, 19) (Fig 1B). Similar patterns were seen in terms of groups of EMT/MET inducers in other EMP networks (Fig S2A). 


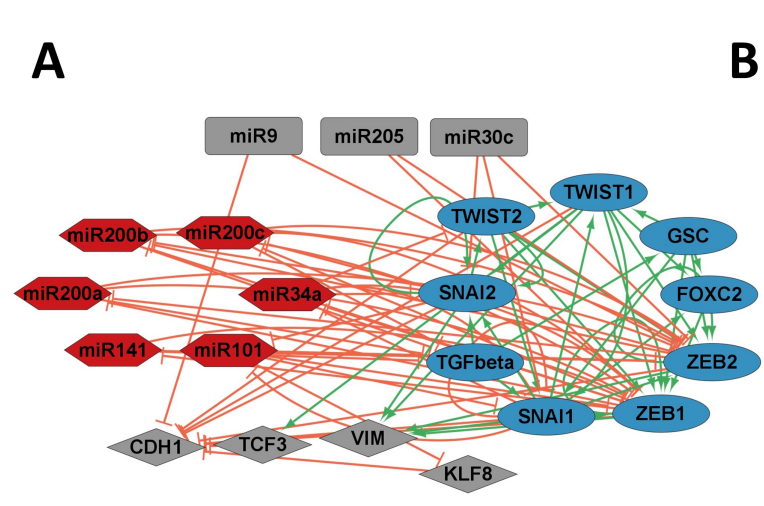

$22 N 82 E$
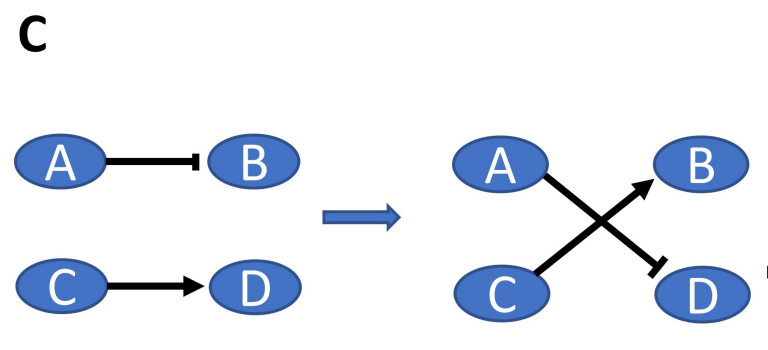

22N 82E

Adjancency Matrix

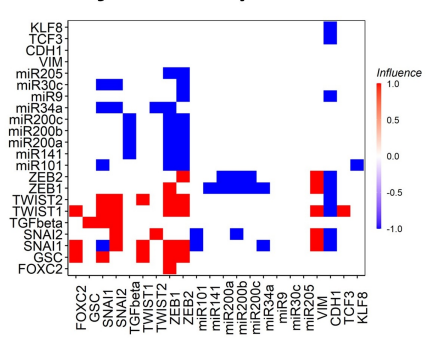

$$
\operatorname{Infl}=\frac{\sum_{l=1}^{\operatorname{lmax}} \frac{A d j^{l}}{\operatorname{Adj} j_{\max }^{l}}}{\operatorname{lmax}}
$$

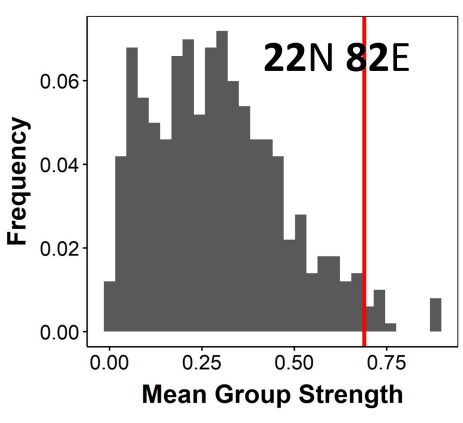

22N 82E

Influence Matrix

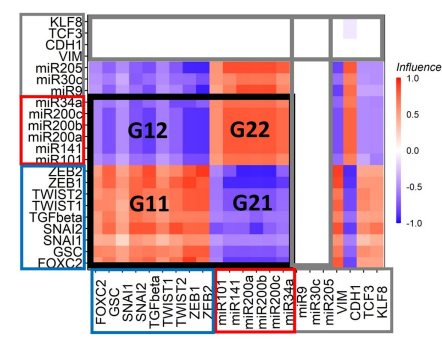

$\begin{aligned} G_{I J} & =\frac{\sum_{i \in I, j \in J} I n f l_{i j}}{n_{I I}}\left|G_{i j}\right| \\ G_{S} & =\sum_{i, j \in\{1,2\}} \mid G_{i j}\end{aligned}$

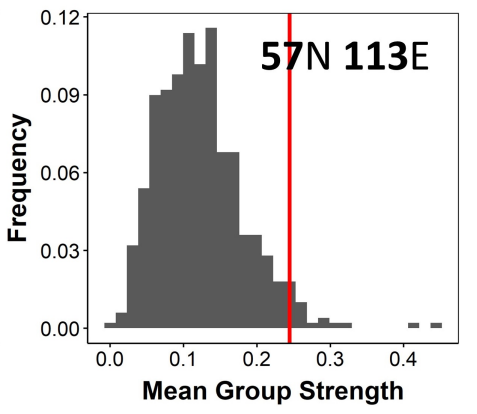

D

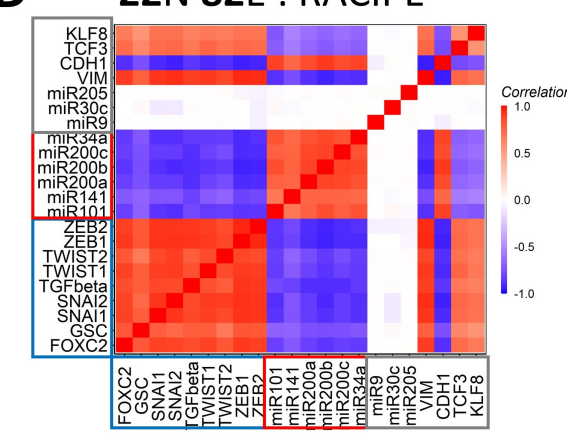

E

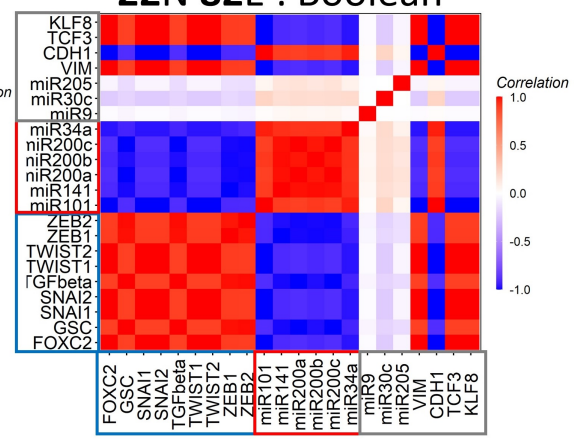

$\mathbf{F}$

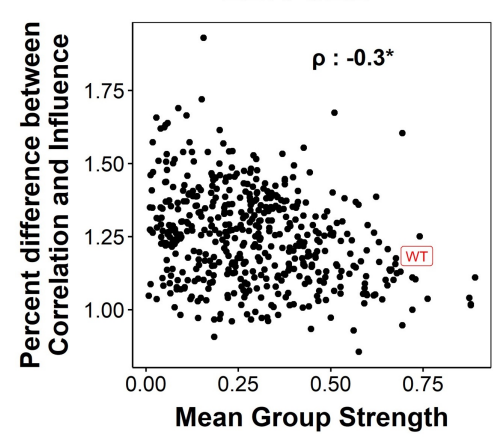

Figure 1: Biological networks show two well coordinated teams (A) EMP network of size 22N 82E, where N stands for number of nodes and $E$ stands for number of edges. (B) The adjacency matrix (left) and influence matrix (right) for the 22N 82E network, with the formula for conversion given below the adjacency matrix. The formula for mean group strength (Gs) is given below the influence matrix. The signal and output nodes (peipheral) are highlighted in a grey box, the mesenchymal nodes (group 1) are highlighted in a blue box and the epithelial nodes (group 2) are highlighted in the red box.. (C) Distribution of mean group strength (Gs) for random networks of size (i) $22 \mathrm{~N} 82 \mathrm{E}$ and (ii) $57 \mathrm{~N} 113 \mathrm{E}$. The Gs values for the corresponding wild-type EMP networks have been highlighted using the red vertical lines. . (D) Correlation matrix for the expression levels of nodes of the 22N 82E network, as obtained by the RACIPE formalism. (E) Same as D but for Boolean. (F) The correlation between the difference between the influence matrix and Boolean correlation matrix (y-axis) and the mean group strength of the network ( $x$-axis) for random networks of size 22N 82E. The wild-type EMP network is highlighted in red. Spearman's correlation coefficient reported. $*: p<0.05$ 
EMP network topology. We then generated 500 hypothetical networks for each EMP network by randomly swapping the edges, ensuring that the in and out degrees of all nodes remain the same, but the way they are connected (network topology) changes $(12)($ Fig $1 \mathrm{C})$. To quantify the existence of distinct groups, we defined a measure of strength of groups in a network: mean group strength (Gs) (Fig 1B, right). A distribution of the group strengths of the hypothetical networks revealed that high mean group strength and therefore the existence of distinct teams of nodes is a property largely unique to the wild-type (WT) EMP network topologies (Fig 1C, S2B).

Next, we wanted to understand the contribution of groups or "teams" of nodes to the emergent properties of the EMP networks. We simulated the dynamics of these networks using two complementary methods: a continuous, parameter agnostic method known as RACIPE (15) and a parameter independent threshold based, asynchronous Boolean formalism (10). The choice for the number of initial conditions to be simulated for Boolean simulations and the number of kinetic parameter sets for RACIPE was made to maintain a balance between minimizing deviation across replicates and minimizing computational time (Fig S2 C-D). We then calculated pairwise correlation between all pairs of nodes across all steady state solutions identified. The correlation matrices of the steady states obtained from RACIPE as well as from Boolean were strikingly similar to one another and showed the same two teams of nodes as that seen in corresponding influence matrix (Fig 1D, S2E-F), suggesting that the "teams" identified in influence matrix (without performing any dynamical simulations) are conserved in the corresponding correlation matrix (identified after simulations). We quantified the distance between the influence matrix and Boolean correlation matrix for the biological network (WT case) as well as the hypothetical networks (see Methods). Intriguingly, the distance between the matrices was lower for the biological networks as compared to that of the hypothetical networks. Also, this distance was negatively correlated with the group strength of a network (Fig 1E, S2G). These results indicate a causative relationship between the existence of team structure of nodes and the emergent dynamical phenotypes of a network. Furthermore, for all networks taken together (WT and hypothetical), the correlation matrix and influence matrix differ by a maximum of $2-3 \%$, suggesting that influence matrix can be a good predictor of the correlation matrix and consequently of diverse phenotypic compositions, irrespective of the strength of teams observed in a given network.

\subsection{Presence of teams of nodes reduces the frustration of phenotypes in EMP networks}

The correlation matrices we saw earlier can be thought of as representative of an ensemble property for a population of cells, each with the underlying biological networks (but with some vari- 
ations in kinetic parameters). Next, we wanted to understand if the team structure also correlates with other properties of the system, specifically, the stability of relevant biological phenotypes. This property is crucial to understand particularly in systems like EMP, where a balance between the ability to switch from a state vs. maintaining it is crucial for robust embryonic development, as well as in establishing cancer metastasis (20).

One way to define the stability of a phenotype (a steady state in the context of network simulations) is the frequency of the steady state (number of initial conditions converging to that steady state). We found that the distribution of steady state frequency (SSF) across all EMP networks is bimodal, with the spread of the unimode corresponding to high steady state frequency being smaller (Fig 2A). It should be noted that the distinction between two modes is the least clear in the $57 \mathrm{~N} 113 \mathrm{E}$ network, which also has a lower mean group strength as compared to the other four EMP networks studied here (Fig S2G). Therefore, we predict that having two strong distinct "teams" can contribute to such bimodal distributions in SSF plots. Our prediction is supported by analysis of hypothetical networks, most of which have a lower group strength than the corresponding WT biological network, also have a lower SSF bimodality coefficient, i.e., a weaker bimodality (21), supporting our prediction (Fig S3A). However, the range of the values of steady state frequencies depends on the size and the density of the networks, among other factors. This dependency makes this metric (SSF) unfit to be used for comparison across networks to identify any generic principles.

Another way to measure the stability of a steady state is its frustration. Frustration can be understood as the conflict between the steady state and the network topology. For a given steady state, an edge is said to be frustrated if the nodes participating in the edge have levels that oppose the nature (sign) of the edge. We calculate the fraction of such edges in the network corresponding to a given state and use that as a metric to measure the stability of that state (16) (Fig 2B, Methods). Similar to the steady state frequency distribution, the frustration values of all states obtained from a given network show high bimodality, unlike most of the corresponding hypothetical (random) networks (Fig 2C, S3B). Further, the higher the frustration of a steady state, the lower is its frequency and vice versa, as observed across EMP networks as well as their hypothetical randomized counterparts (Fig 2D, S3C), supporting a generic behavior across these metrics.

Intriguingly, the maximum frustration value seen across steady states for all EMP networks (WT as well as random) is approximately 0.45 (Fig 2D). To identify the patterns of frustration metric in WT networks vs. their randomized counterparts, we compared the corresponding minimum 
and maximum frustration values as obtained across states for a given network. The minimum frustration value of the biological networks (i.e., most stable states) was found to be lower than most of the corresponding values obtained for random networks (Fig 2E). However, the same is not true for maximum frustration or mean frustration (Fig 2E). With the exception of the 22N82E network, $50 \%$ or more of corresponding hypothetical networks can have mean and/or maximum frustration values, but not minimum frustration values, lower than those noted for the biological networks (Fig 2E, ii). Furthermore, the hypothetical networks show an increased number of steady states than the WT EMP networks (Fig 2E, ii). Together, these results suggest that the EMP network topologies are designed to support the less frustrated (highly stable) states.

Frustration, being the measure of conflict between a state and the network topology can have a negative effect of stability of the state, i.e., the lower the frustration, the higher is the expected stability of the state. Thus, in Boolean simulations, where the dynamics are determined by the network topology alone (no kinetic parameters), we observed a strong negative correlation between the frustration and steady state frequency (Fig 2D). Next, we calculated frustration and its correlation with state frequency using the discretized steady states obtained from RACIPE. The correlation between frustration and steady state frequency for all WT networks as obtained for RACIPE was weaker than that of Boolean simulations (Fig 2F, S3D). This weakening can be attributed to additional low-frequency (and high-frustration) steady states obtained via RACIPE, as also witnessed for the SCLC network earlier (12). Having identified a negative correlation of frustration with steady state frequencies obtained using either RACIPE and Boolean simulations, we next investigated if the mean group strength of a network affects the frustration of the steady states for the corresponding network. We observed that the minimum frustration value of a steady state of a network, but not the maximum frustration, negatively correlates with the corresponding mean group strength, as observed for 22N82E network and its corresponding hypothetical networks (Fig 2G, i-ii). This trend was consistently demonstrated in EMP networks of all sizes considered (Fig $\mathbf{2 G}$, iii), suggesting that the presence of strong groups or "teams" promote the existence of highly stable and less frustrated phenotypes. 
A

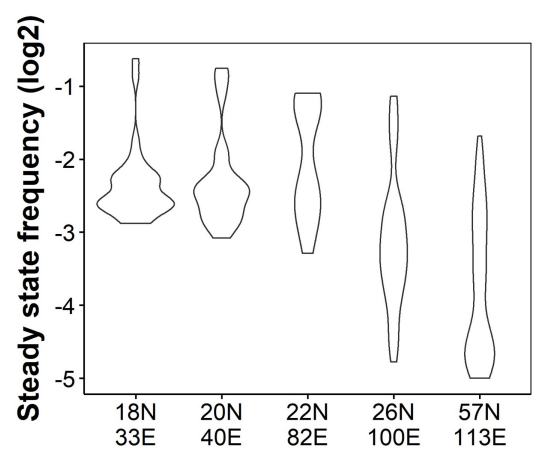

D

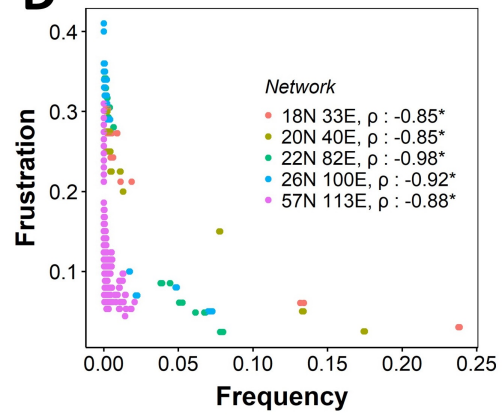

B

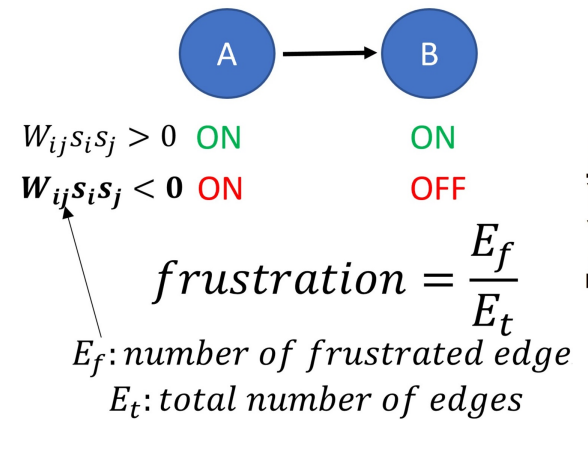

C

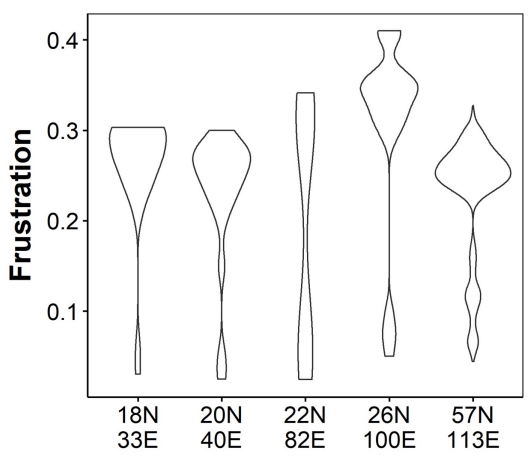

E ii)

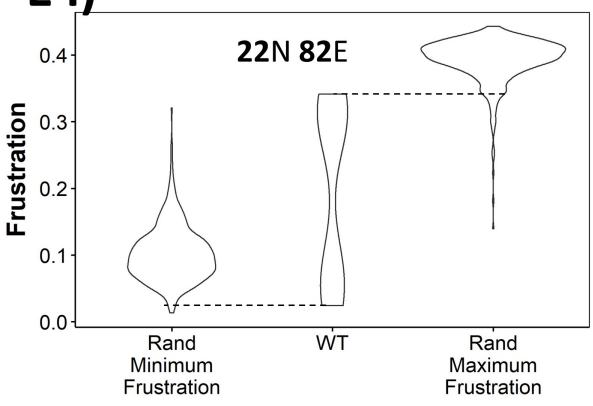

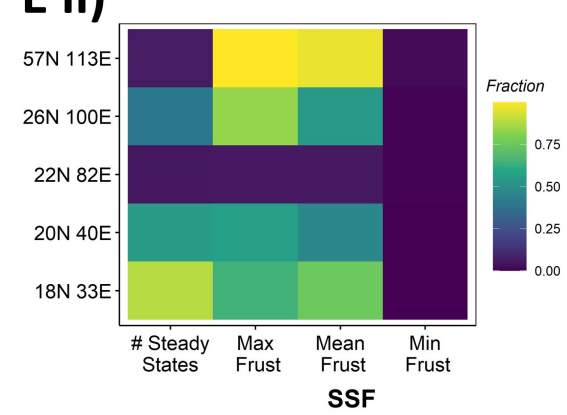
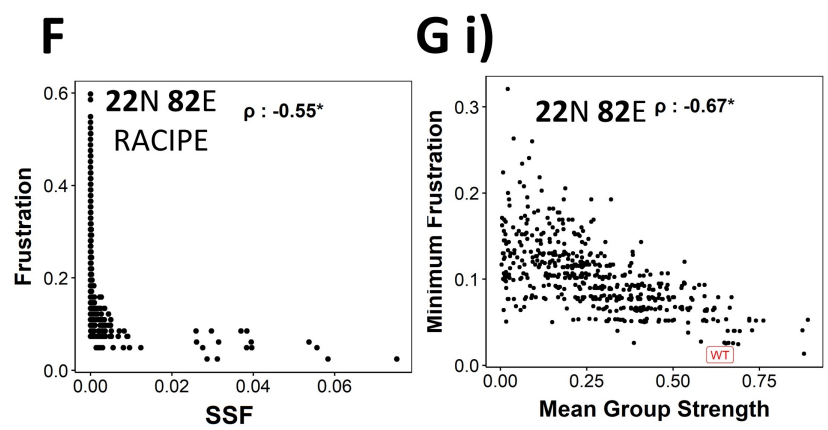

G ii)

G iii)

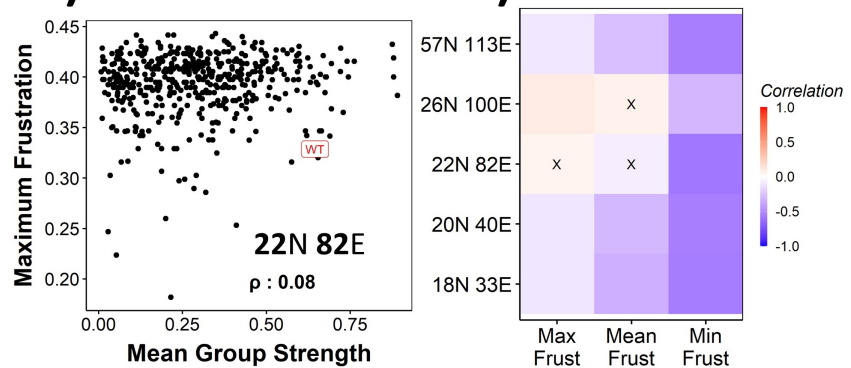

Figure 2: Presence of teams reduces the frustration of EMP phenotypes (A) Violin plots of the distribution of steady state frequencies obtained from Boolean simulations of different EMP networks. (B) Depiction of frustration calculation of a state. (C) Violin plots of the frustration of steady states obtained from Boolean simulations of different EMP networks. (D) Scatterplot of Frustration vs steady state frequency for all EMP networks obtained via Boolean. Corresponding spearman correlation coefficient is reported, $*: p<0.05$. (E) (i) Comparison of the distribution of frustration for the WT EMP network (22N 82E), with the distribution of maximum frustration values and minimum frustration values of corresponding random networks. (ii) Heatmap showing the fraction of random networks that show a higher number of steady states (column 1) or higher frustration metric (columns 2-4) as compared to the WT EMP networks. (F) Scatterplot of Frustration vs steady state frequency for 22N 82E EMP network obtained via RACIPE. Corresponding spearman correlation coefficient is reported, $*: p<0.05$. (G) Scatterplot of minimum frustration (i) and maximum frustration (ii) of random networks of size 22N 82E with the mean group strength. Corresponding WT network is labelled and the spearman correlation coefficient has been reported, $*: p<0.05$. (iii) Spearman correlation between frustration metrics and Gs for random networks of all sizes , $X: p>0.05$. 


\subsection{Presence of teams increase the dynamic stability of EMP phenotypes}

Both SSF and frustration are static metrics, and do not provide any information about the dynamics of the steady states upon fluctuations in the environment. Because plasticity is a crucial aspect of EMP phenotypes, understanding the perturbation dynamics is crucial for EMP networks. To capture the dynamical stability of phenotypes, we defined another metric that we term coherence, inspired from a similarly designed metric (22). This metric measures the fraction of times a given steady state returns to itself, when subjected to dynamical perturbations, i.e., change in the level of one or more of the nodes in a network (Fig 3A). The coherence of a state can hence vary between 0 and 1 , with 0 indicating the least stable steady state and 1 indicating a highly stable steady state from a dynamical perturbation view. Therefore, one way to interpret coherence is the fraction of neighbour states of a steady state that belong to the basin of attraction of that steady state. Here, for coherence calculations, we apply the smallest possible dynamical perturbations to the steady state (changing the value of one node from 1 to 0 or vice versa); examples of stronger perturbations including multi-node perturbation, time-varying noise etc.

Similar to our observations in frustration and SSF, coherence also shows a bimodal distribution for all EMP networks. The bimodality coefficient for WT EMP networks is higher than most of the corresponding random networks (Fig 3B, S4A). Also the maximum coherence value seen in WT network is larger than those noted for most corresponding random networks, but this trend is not seen for the minimum coherence values (Fig 3C). Furthermore, the maximum coherence, but not minimum coherence, of networks correlates positively with their mean group strength, as noted across a cohort of a WT (22N82E) network and its random counterparts (Fig 3D i,ii). This trend is seen for other networks as well (Fig 3D, iii), endorsing our previous conclusions that the presence of strong "teams" (as measured by mean group strength) supports the existence of highly stable states. 

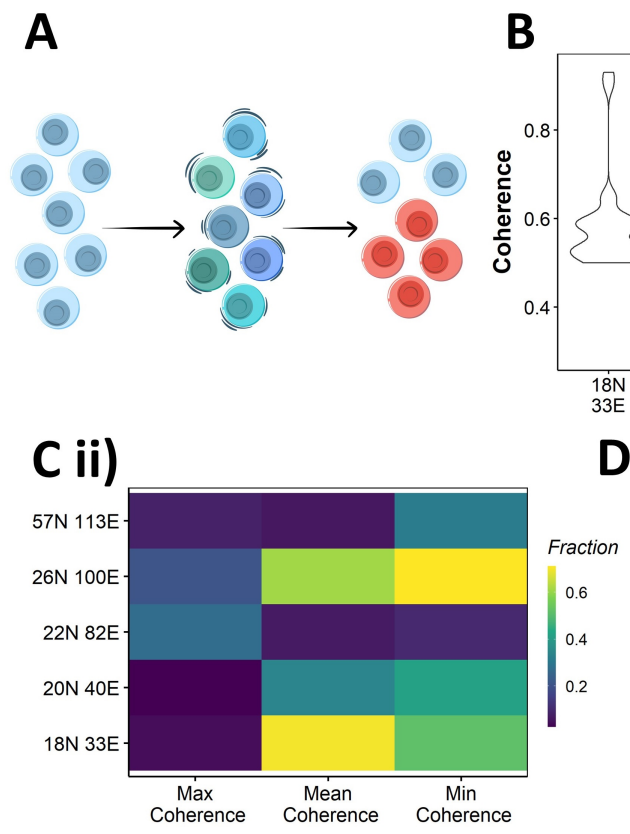

D iii)

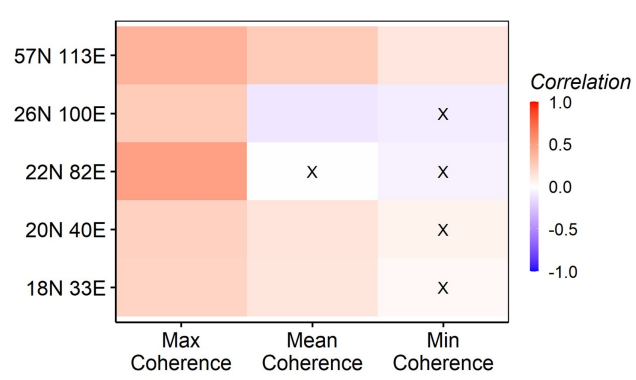

B

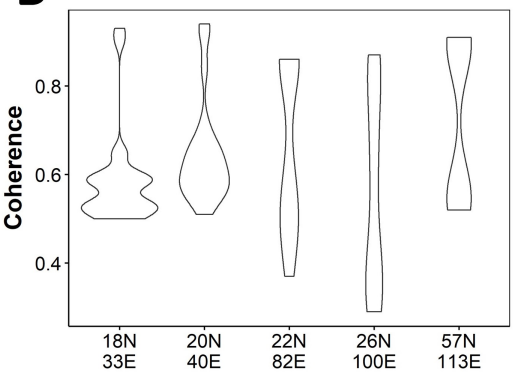

Ci)

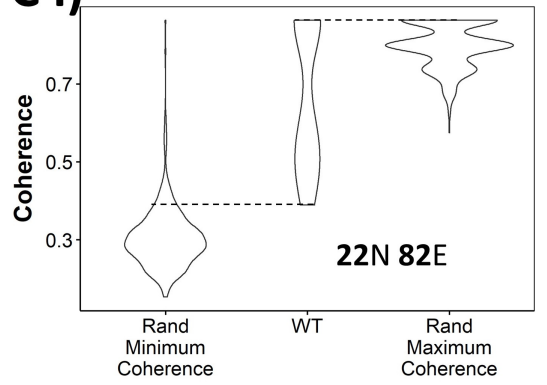

D ii)

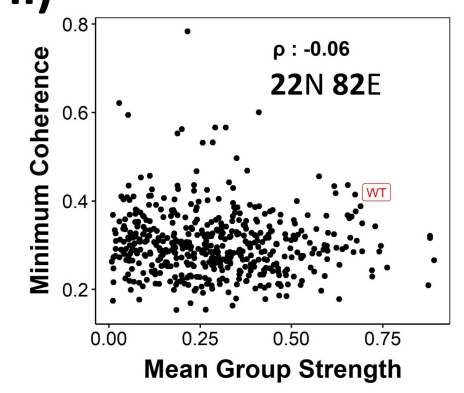

E

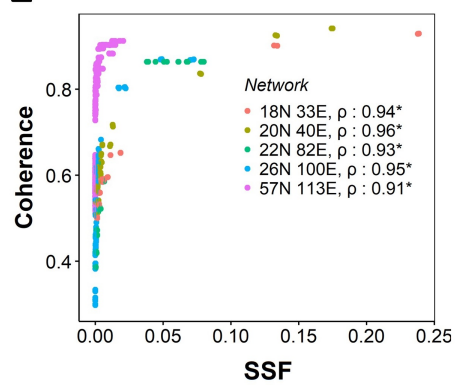

$\mathbf{F}$

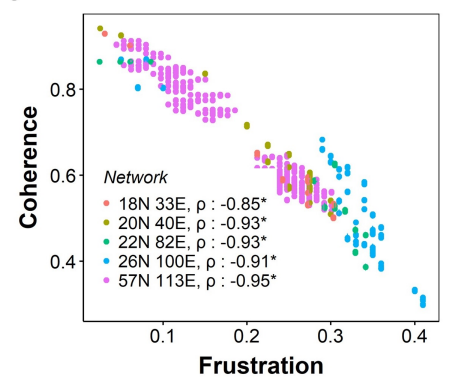

Figure 3: Presence of teams increases the coherence of EMP phenotypes (A) Depiction of coherence metric a measure of the probability that a steady state is re-attained after single node perturbation. (B) Violin plots of state coherence for the WT EMP networks. (C) (i) Comparison of WT state coherence distribution with the distribution of maximum coherence of random networks (right) and minimum coherence of random networks (left) for networks of size $22 \mathrm{~N} 82 \mathrm{E}$. (ii) Heatmap depicting the fraction of random networks having higher coherence metrics than the corresponding WT EMP networks. (D) Scatterplot showing the correlation between Mean Group Strength (Gs) of the random networks of size $22 \mathrm{~N} 82 \mathrm{E}$, with the maximum coherence (i) and minimum coherence (ii) of the networks. WT is labelled in red. (iii) Correlation between Gs and coherence metrics for random networks derived from the five EMP networks, $X: p>0.005$. (E) Scatterplot of coherence and frequency of the steady states of WT EMP networks. The Spearman correlations are reported in the legend. (F) Same as D, but for Coherence and Frustration of steady states.

We find that coherence values of steady states are positively correlated with their frequency, as expected (Fig 3E), because high coherence implies a larger attractor basin and hence higher frequency. The correlation was stronger for WT networks as compared to the hypothetical networks, indicating a role of "teams" in strengthening these correlations (Fig S4B). Coherence of a steady state is also negatively correlated with its frustration; again, the correlation is stronger for 
WT networks than for corresponding hypothetical ones (Fig 3F, S4C). Importantly, the coherencefrustration scatter plot unravels a clear presence of the two groups of states arranged diagonally - (high frustration, low coherence) and (low frustration, high coherence); the former are more dynamically stable than the latter ones (Fig 3F).

\subsection{Terminal and Hybrid phenotypes in EMP networks}

We noted remarkably consistent characteristics among different stability metrics for diverse EMP networks. Particularly, all of them showed bimodality in their distributions, suggesting that there are two types of steady states observed in EMP networks. A two-dimensional plot marking the values for all stability metrics of the steady states (coherence, frustration, steady state frequency) clearly differentiated between these two groups of states (Fig 4Ai, S5A). To test if these groups of states depend on the mean group strength, we took 20 hypothetical (random) networks corresponding to $22 \mathrm{~N} 82 \mathrm{E}$ network - 10 of them with the maximum mean group strength (Gs) and 10 of them with the minimum Gs - and plotted their steady states on the coherence-frustration plot. We see that while the high Gs networks show a clearly bimodal set of states - those with (high coherence, low frustration) and with (low coherence, high frustration), the low Gs networks show a more scattered pattern of states, as shown by their lower bimodality coefficient in this plot (Fig 4A,ii; S5B). This result suggests that strong "teams" are more likely to give rise to two distinct sets of states with different stability features.

Next, we analysed the biological nature of these sets of steady states in WT networks. The group of states having (high frequency, high , coherence, low frustration) could be further classified into two sub-groups, based on the common nodes that are upregulated or downregulated in these states (Fig 4B, S5C). The first sub-group showed consistently high levels of all epithelial markers/MET inducers and low levels of all mesenchymal markers/EMT inducers, while the second sub-group had an exactly opposite pattern. Thus, these states can be mapped on to epithelial and mesenchymal phenotypes respectively. On the other hand, the other set of states the ones with (low coherence, low frequency, high frustration) - had a mixed expression pattern of many epithelial and mesenchymal nodes; thus, these states can be mapped on to various hybrid epithelial/mesenchymal phenotypes.

Importantly, the two sets of epithelial and mesenchymal nodes seen in the two sub-groups are identical to the two "teams" identified from the influence matrix without any dynamical simulations (Fig 1B, S2A). The levels of input/output (peripheral) nodes do not show any consistent patterns across the states observed (see the nodes highlighted in grey box in Fig 4B). Put together, three 
classes of biological phenotypes emerge from the EMP networks: epithelial, mesenchymal and hybrid, agreeing with various experimental observations $(23,24)$. Further, each of these three phenotypes (macro-states) is composed of multiple "micro-states" with a common core expression pattern.

To investigate the properties of these different phenotypes, we defined an EMT score for states using the expression values of the core epithelial and mesenchymal nodes, varying between -1 and 1, such that the epithelial states have an EMT score of +1 , the mesenchymal states have an EMT score of -1 and the hybrid states have a score between -1 and 1 . Classifying the steady states of RACIPE based on the EMT score lead to consistent steady state patterns with those seen in Boolean, i.e Epithelial and Mesenchymal states have a higher SSF than the Hybrid states (Fig 4C, S6A). Hence, biologically, the states emergent from the EMP networks can be categorized into three classes based on the EMT score, while in terms of stability, they can be categorized into two classes: Terminal (Epithelial and mesenchymal phenotypes clubbed together) and hybrid states. In conclusion, the topology of various EMP networks enables the existence of terminal phenotypes (epithelial, mesenchymal) which are more frequent and coherent but less frustrated, and hybrid epithelial/mesenchymal phenotypes that are less frequent and coherent but are more frustrated.

\subsection{Teams of nodes stabilize terminal phenotypes}

Given the correlation between Gs and stability metrics, as well as the strong similarity between influence and correlation matrices, we wanted to check if the influence matrix alone can explain the stability of terminal and hybrid states of a network, without necessarily requiring the presence of teams. teams. In other words, can the influence matrix be used to predict the frequency of individual states/phenotypes emerging from a network, in addition to predicting a phenotypic distribution from a network. To investigate this aspect, we defined the strength of a state based on the influence matrix ( Fig 4, Methods). As expected, the strength of terminal states is much higher than the strength of the hybrid states in all EMP networks in both RACIPE (Fig 4Di, S6B) as well as in Boolean simulations (Fig 4Dii, S6C). The random networks, however, showed no such distinction between the distribution of state strength of Hybrid and Terminal phenotypes when the phenotypes were defined using the strongest "teams" possible in corresponding random networks (Fig 4D,iii; S6C). 
A i)

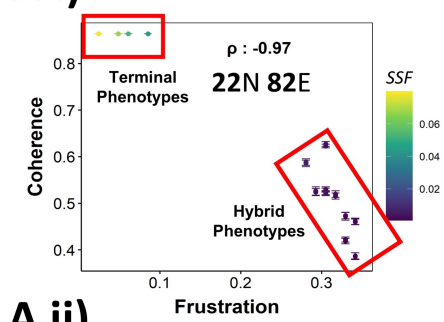

A ii)

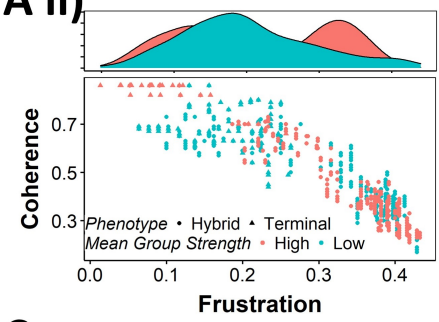

C

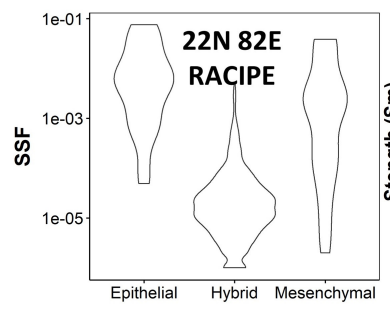

E i)

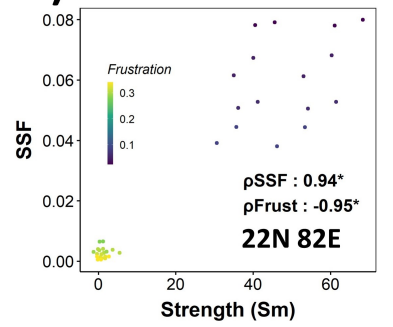

B

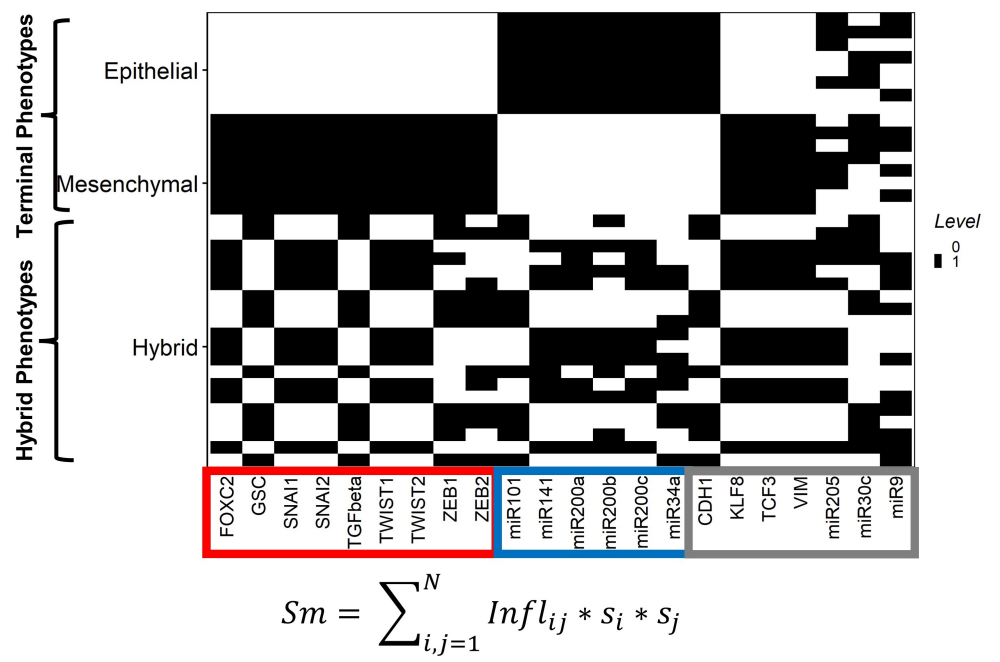

D ii)

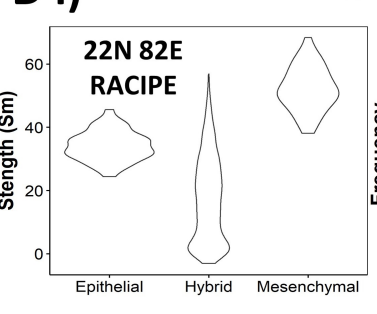

E ii)

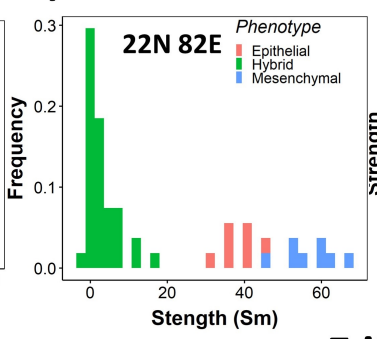

E iii)

D iii)
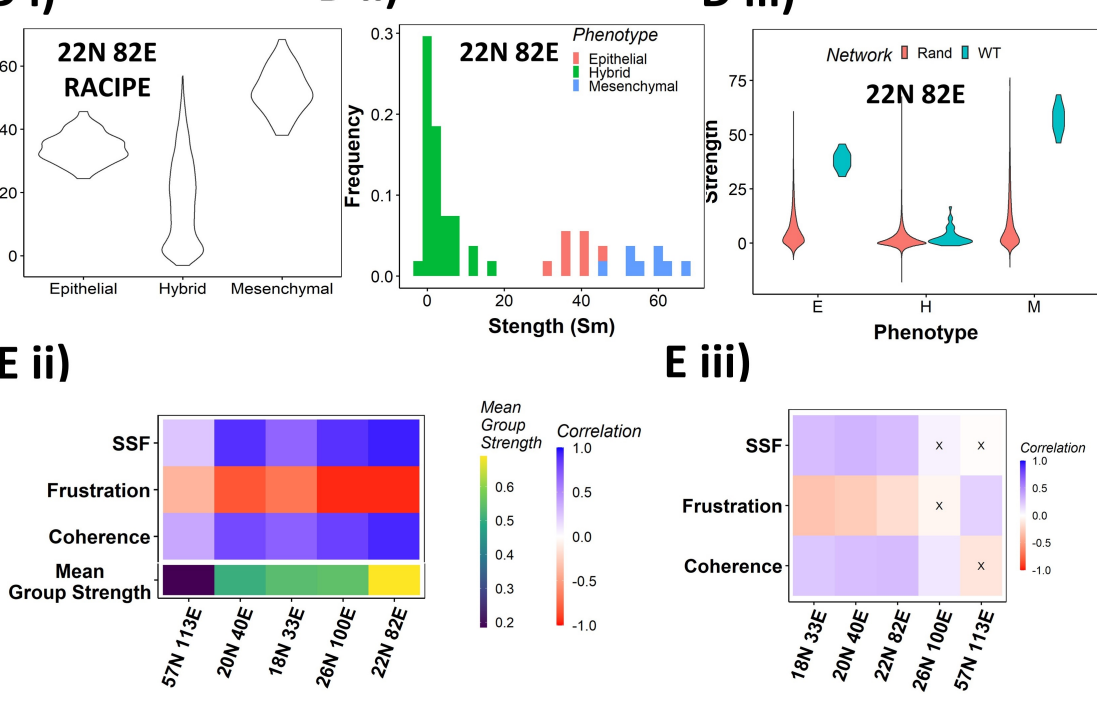

Figure 4: Teams of nodes enable terminal phenotypes (A)(i) Frustration, Coherence and SSF for the steady states of WT EMP network of 22N 82E. Two classes of phenotypes (terminal and hybrid) obtained based on their stability have been labelled. The correlation between frustration and coherence is reported on the network. (ii) Scatter plot showing the states of top 10 and bottom 10 (based on mean group strength) random networks of size $22 \mathrm{~N} 82 \mathrm{E}$. The density distribution is for the composite axis of coherence + frustration. (B) Heatmap depicting the steady states of 22N 82E WT EMP network. The epithelial, mesenchymal, and peripheral (signal and output) nodes, as identified from corresponding influence matrix, have been highlighted with red, blue and grey rectangles respectively. (C) Distribution of SSF for the RACIPE states of 22N 82E WT EMP network. The state classification is done based on teams identified in B. (D) Distribution of the strength of states of the 22N 882E network (i) from RACIPE and (ii) from Boolean simulations. (iii) Comparison of the state strength distributions for Epithelial, Mesenchymal and Hybrid phenotypes obtained from WT and random networks of size 22N 82E. State classification for random networks was done by clustering their corresponding influence matrix. Among the two sets of terminal states, that states having the highest number of micro-RNAs turned "on" were classified as the Epithelial states. The formula for calculation of state strength is given above the panel. (E) (i) Correlation of state strength with SSF and Frustration for 22N 82E WT network. (ii) Correlation between the stability metrics ( $y$ axis) and the strength for the states of the five different WT EMP networks ( $x$-axis). The mean group strengths of the networks is represented using a different color scheme at the bottom of the heatmap. (iii)Correlation between the quantities in $E$ (ii) and $G$ s for random networks of corresponding to WT networks of different sizes $X: p>0.05$. 
The state strength also correlated with different state stability metrics for all EMP networks: positively with frequency and coherence, and negatively with frustration (Fig 4E, i-ii). Interestingly, we observe that when compared across EMP networks, the extent of the correlation of state strength with stability metrics decreases with the decreasing Gs of a network (Fig 4E,ii). These results suggest that the presence of strong teams of nodes in the network is crucial for terminal phenotypes supported by these teams. To confirm this trend, we calculated the correlation between the state strength and stability metrics for hypothetical (random) networks corresponding to different EMP networks. Again, the higher the value of Gs, the stronger the correlation between state strength and stability metrics (Fig 4E, iii). Put together, these analyses suggests that the state strength as computed from the influence matrix alone cannot explain the enhanced stability of the terminal phenotypes, but the presence of strong "teams" of nodes can.

\subsection{Teams structure leads to robust terminal phenotypes}

Having identified that the presence of "teams" of nodes that cooperate with/activate each other can reinforce a given steady state when a perturbation to one of the node values was made (coherence), we next asked whether the positive reinforcement in a network offered by "teams" can be extended to a scenario when more than one node is perturbed. First, we took a closer look at the coherence metric by analysing the coherence patterns of the steady states when each node of the network is perturbed one at a time (Fig 5A, S7A). As expected, the terminal phenotypes remained unchanged when any of the core nodes was perturbed. But the hybrid phenotypes had a relatively smaller coherence for such cases. Upon perturbing some of the signal nodes, however, we noticed that the hybrid as well as terminal phenotypes showed a coherence of 0 . Upon close inspection at the dynamics of the perturbation, we found that for terminal states, the perturbation in signal nodes does not change the configuration of the core nodes. However, because the signal nodes do not have any input to them, perturbation of the signal nodes does not revert over the course of the simulation. Similarly, output nodes are always restored back upon perturbation without any effect on the steady state. Next, we considered the case of perturbing multiple nodes (randomly chosen) simultaneously and calculated the coherence of the steady states. While the terminal states show resilience up to $25 \%$ of nodes being perturbed, the hybrid states lose their identity (i.e. switch to another state) upon minor perturbations (Fig 5B, S7B). 
A

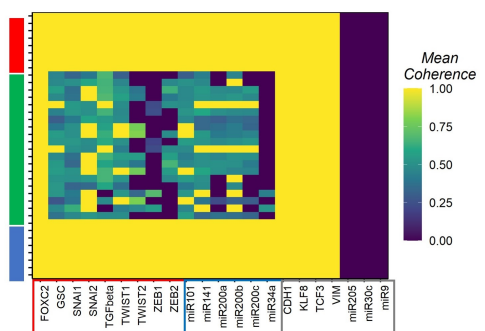

B

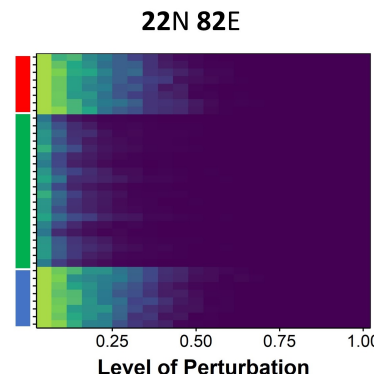

C

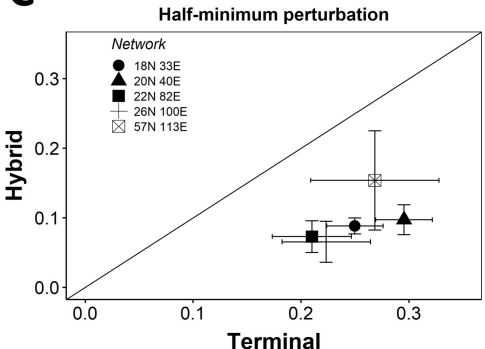

\section{D i)}

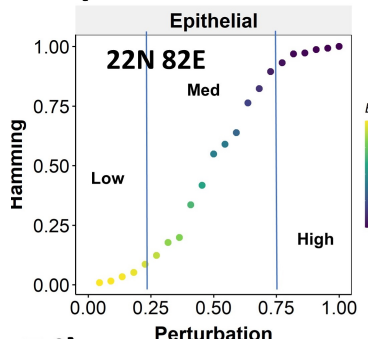

Ei)

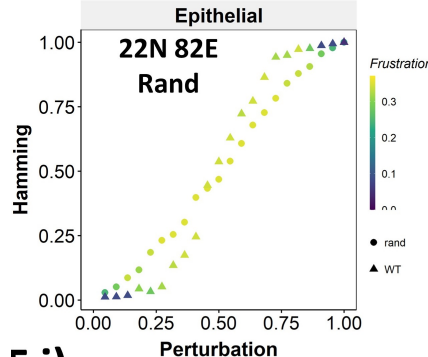

$\mathrm{F}$ i)

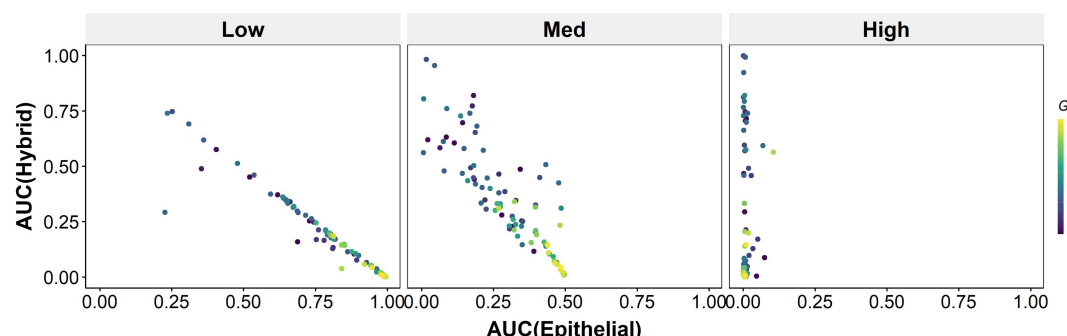

G i)

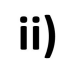

ii)

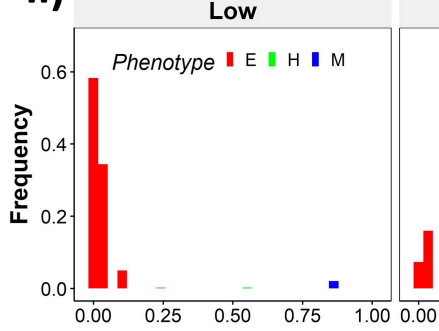

ii)
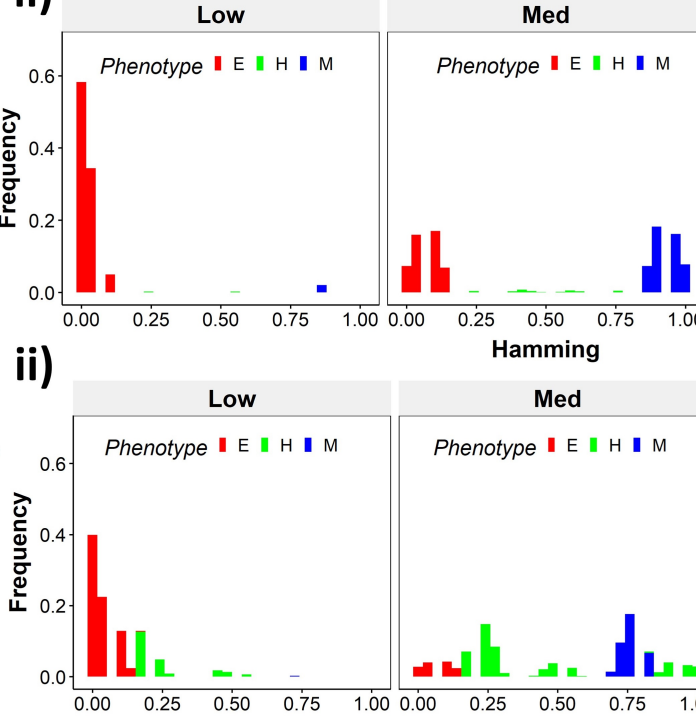

Med

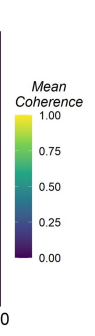

Low

Med

High

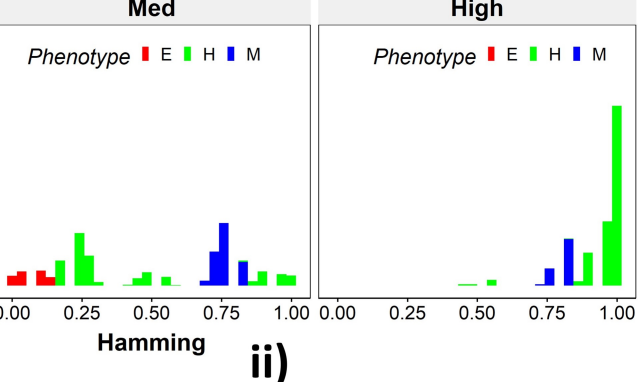

ii)

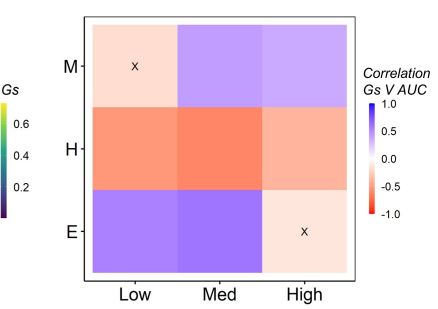

ii)
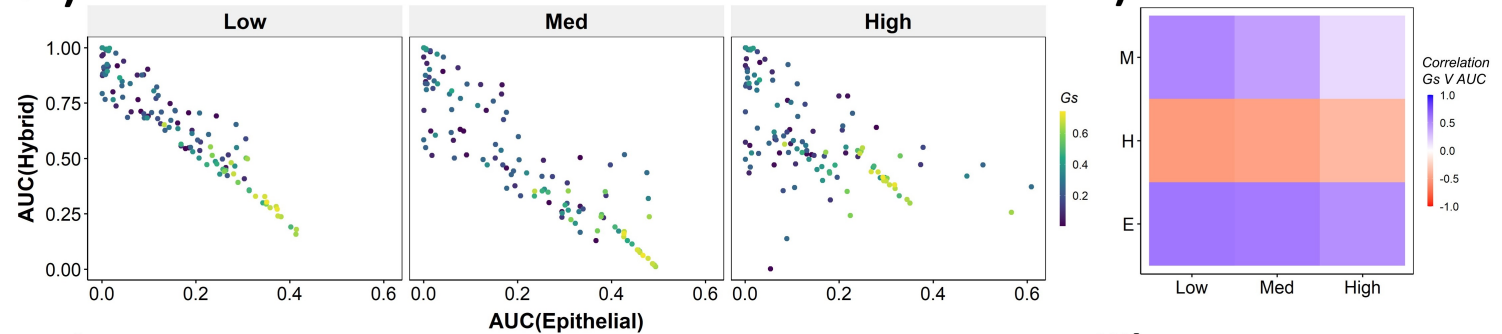

iii)

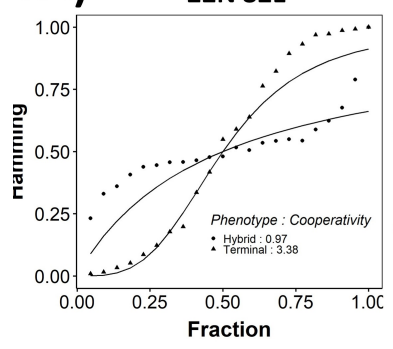

ii)

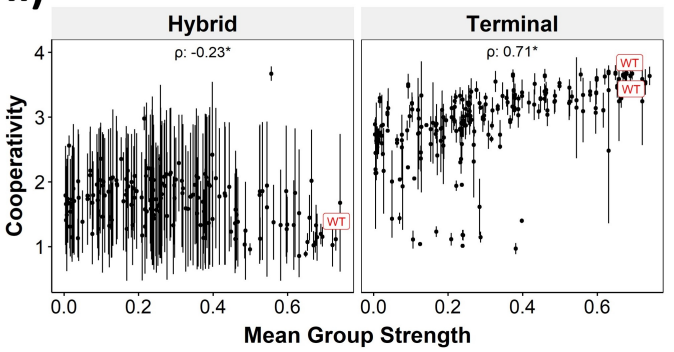


Figure 5: Teams of nodes enable terminal phenotypes (A) Heatmap depicting the mean coherence of the Boolean states of 22N 82E WT EMP network when each node is individually perturbed. (B) Mean coherence of states of $22 \mathrm{~N}$ 82E EMP network with multiple nodes perturbed at once (Level of perturbation). (C) Extent of perturbation required to bring the coherence of terminal phenotypes ( $x$-axis) and hybrid phenotypes ( $y$-axis) below $0.5 . x=y$ line is shown. (D) (i) Representative mean Hamming distance plot of an epithelial state obtained from 22N 82E WT network. Three levels of perturbation are highlighted based on regions of the sigmoidal plot. (ii) Distribution of the Hamming distance from the starting state in D (i) at different levels of perturbation, colored by the phenotype. (E) (i) Representative mean Hamming distance plot comparing the dynamic transition of an epithelial state from WT and that from a random network. (ii) Corresponding distribution of Hamming distances for the random network. (F) (i) Scatter plot between mean AUC of Epithelial states and mean AUC of hybrid states when starting from epithelial phenotype. Each dot is a random network, colored by its mean group strength. (ii) Heatmap depicting the spearman correlation between mean group strength and AUC for the final phenotype (y-axis) and the regions in the sigmoidal plot (x-axis). (G) Same as F, but for Hybrid as the starting phenotypes. (H) (i) Depiction of cooperativity with a terminal and hybrid state of the WT EMP network 22N 82E. (ii) Mean group strength vs cooperativity for random and WT networks of size 22N 82E. Each dot represents the mean cooperativity for one network. The bars show standard deviation. (iii) Correlation between group strength and cooperativity for random networks corresponding to WT EMP networks of different sizes.

We quantified this difference in the EMP networks by measuring the extent of perturbation at which the coherence goes below 0.5 (termed as Half-minimum perturbation) for the terminal as well as hybrid states (Fig $\mathbf{5 C}$ ). As expected, the half-minimum value for the terminal states is always higher than the same for the hybrid states (all 5 EMP networks lie below the $x=y$ diagonal in Fig 5C). Interestingly, the 57N113E network that had a lower mean group strength among the EMP networks showed slightly higher resilience for hybrid states as compared to the other EMP networks, suggesting that as the strength of teams decreases, the difference between hybrid and terminal states in their stability tends to reduce. This argument is further strengthened by analysis of random networks (with Gs often less than that in WT networks) showing a comparatively higher resilience of hybrid states (as visualised by the distance from the $x=y$ diagonal) (Fig S7C). Put together, these results endorse that "teams" of nodes can also play a crucial role in maintaining a terminal phenotype, even when multiple nodes within a network are disrupted.

\subsection{Teams structure imparts unique transition characteristics to Hybrid phenotypes}

Given the clear difference in the dynamical stability of hybrid and terminal phenotypes, we wanted to understand the biological implications of the dynamical stability characteristics and the presence of "teams". The multi-node perturbation experiment in Fig 5B can be perceived as a state transition- inducing signal. The higher the extent of perturbation, the stronger the signal. Given this interpretation, we interrogated whether the transition trajectory/characteristics depend on the presence of teams in the network. To better quantify this dynamics, we performed an experiment mimicking a population of 100 cells exposed to a transitory signal. Each cell in the population starts from one steady state, and a random perturbation of a certain extent (varying from 0 to 1 ; i.e., no node from perturbed to all nodes being perturbed together) is given to each cell. The state 
of the cell is then allowed to evolve until a steady state is reached. We repeat this experiment 10 times for each steady state of a network and perform statistical analysis. To measure the phenotypic transition, we made use of the Hamming distance between the initial (unperturbed) steady state and the steady state obtained after perturbation. The Hamming distance between two states is calculated as the fraction of nodes having different expression level between the two states. The Hamming distance varies between 0 and 1, where a Hamming distance of 0 indicates identical states and a Hamming distance of 1 indicates states with all nodes having opposite levels of expression. . States belonging to the same biological phenotype will be separated by relatively smaller Hamming distance (close to 0 ), due to the presence of "teams".

The terminal phenotypes of EMP networks show a sigmoidal transition curve in terms of the mean Hamming distance across all replicates (Fig 5D, i). In other words, at low levels of perturbation, the Hamming distance and change in EMT score remains low, suggesting that "teams" offer "resistance" to the signal, leading to a minimal change in corresponding phenotype. Similarly, at high levels of perturbation, we see a complete change in the phenotype, as measured by the Hamming distance (being close to 1) and a drastic change in the EMT score. At intermediate levels of perturbation, a near linear transition of phenotype is seen. We further quantified the phenotypic distribution (\% of states corresponding to $\mathrm{E}, \mathrm{H}$ and $\mathrm{M}$ phenotypes) in these three levels of perturbation ( as demarcated in Fig 5D,i). As expected, for a population of cells starting from Epithelial phenotype, at low level of perturbation, a majority of the cells remain epithelial, with a very small fraction converting to mesenchymal. At moderate levels of perturbation, we see an equal fraction of cells in epithelial and mesenchymal phenotypes, with a minor fraction switching to hybrid. At high levels, almost all cells turn mesenchymal. To identify how unique this sigmoidal pattern is to an EMP network (containing "teams" of nodes), we evaluated this transition trajectory for a hypothetical/random network with low mean group strength (Fig S7D). Unlike the case with EMP networks, the distinction between terminal and hybrid phenotypes in terms of their transition characteristics mostly disappears and all the phenotypes have near-linear transition characteristics when perturbed (Fig 5E,i; S7E,i-ii). perturbations. Consistently, t he corresponding phenotypic distributions at low, medium and high levels of perturbations look comparable, irrespective of the initial phenotype (Fig 5E,ii; S7E,iii). This difference between the behavior of WT networks and random networks indicates that the teams govern transition trajectories emanating from various terminal or hybrid phenotypes in a network.

To better understand the dependence of the transition dynamics on the mean group ("teams") 
strength, we quantified the area under the curve (AUC) for phenotypic distributions for various levels of perturbations (for panels shown in Fig 5D,ii and 5E, ii). When starting from an Epithelial phenotype, the networks with high group strength show low frequencies of the hybrid states, as compared to the networks with low group strength, irrespective of the degree of perturbation made (Fig 5Fi), suggesting that the presence of "teams" does not enhance the frequencies of hybrid states which may be therefore metastable. A quantification across the epithelial states for random networks as well revealed a negative correlation of the hybrid AUC with group strength at all levels of perturbation (Fig 5Fii). The correlation is positive with mesenchymal AUC at high perturbation and positive with epithelial AUC at high perturbation, as expected, since these are the dominant final (terminal) states in each case respectively. Similar trends were seen for cells starting from mesenchymal phenotypes (Fig S7F).

The AUC analysis for cases with hybrid states as the initial conditions revealed similarly consistent trends. At low and high levels of perturbation, as group strength of the network increases, the AUC of epithelial approaches 0.4 and that of hybrid approaches 0.1 . At medium levels of perturbation, epithelial AUC nears 0.5 and hybrid AUC nears 0 for maximum group strength network (Fig $5 \mathbf{G i}$ ). Unlike the trajectories seen for terminal phenotypes, because there is no preferred phenotype here in terms of either epithelial or mesenchymal, group strength correlated positively with both epithelial and mesenchymal AUCs and negatively with hybrid AUC (Fig 5Gii). These trends between the AUC and group strength were consistently seen across networks of all sizes (Fig S7G). Together these observations indicate that the presence of two teams - one composed of epithelial master regulators and the other composed of mesenchymal master regulators - may reduce the frequency and stability of hybrid E/M phenotypes.

While the AUC analysis provided a good understanding of the transition properties, the mean Hamming distance plots intuitively demonstrated the difference in dynamical characteristics between the terminal and hybrid phenotypes of WT networks and that of random networks well. Hence, we quantified the sigmoidal nature of these transition curves, by fitting them to a simple hill function and calculating the coefficient of cooperativity, $n$, for each such fit (Fig $5 \mathrm{H}, \mathrm{i})$. We then compared the mean cooperativity coefficient for trajectories obtained for terminal phenotypes and hybrid phenotypes of all random and WT networks against the mean group strength of the networks. The cooperativity coefficient of terminal phenotypes increased with increasing group strength, while that estimated for hybrid phenotypes decreased with increasing group strength (Fig $\mathbf{5 H}, \mathbf{i i})$. The correlation of the mean cooperativity coefficient corresponding to terminal pheno- 
types with that of mean group strength was quite consistent across networks of different sizes as compared to that of hybrid phenotypes (Fig $\mathbf{5 H}$, iii). The higher the value of $n$, the more step-like the corresponding sigmoidal function is. Thus these results endorse that the presence of teams makes the transition dynamics highly non-linear, and confers initial "resistance" to exit a terminal phenotype (lag phase of sigmoidal curves).

Therefore, using the random networks as case studies, we were able to establish that the "teams" structure supports the terminal phenotypes dynamically and leads to unique dynamical transition signatures of terminal and hybrid phenotypes in these different EMP networks.

\subsection{Targeted reduction of group strength in a network can reduce stability and robustness of EMP phenotypes}

So far, we have established the connection between the "teams" structure and stability of terminal phenotypes in a correlative manner, across multiple EMP networks of varying sizes. To propose a causative connection, we performed edge deletion experiments with the WT EMP networks. In a given network, we ranked different edges in terms of their deletion being able to maximize a reduction in mean group strength after deleting that edge. Sequential deletion of edges in this manner was performed to bring down the mean group strength to low values (Fig 6 A, i). The networks thus obtained saw a reduction in the stability of the terminal phenotypes, as measured by minimum frustration (Fig 6 A, ii), maximum coherence (Fig 6A,iii) and the frequency of terminal phenotypes (Fig 6A, iv). The dependence of the stability of terminal phenotypes is especially prominent in the areas corresponding to the initial linear regime (number of edges deleted < 5 in Fig 6A,i) where the mean group strength falls sharply. The e stimated cooperativity coefficient ( $\mathrm{n}$ in Hills function) of the transition dynamics of terminal and hybrid phenotypes also showed expected trends: the higher the group strength, the higher the cooperativity (the more sigmoidal the curve) (Fig 6B) . A summary of the correlations obtained between Gs and various metrics is given as a heatmap in Fig 6D. These results suggest that as long as the structure of "teams" is maintained, terminal phenotypes will remain dominantly stable, and their stability can be predicted by the strength of "teams" which can be calculated from the influence matrix alone, without performing any dynamical simulations. 


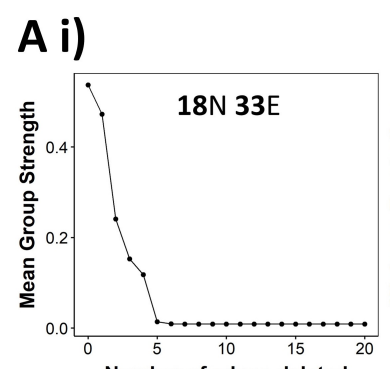

A iii)

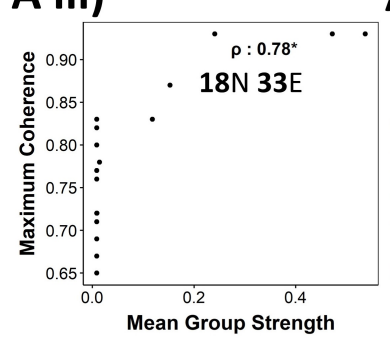

A ii)

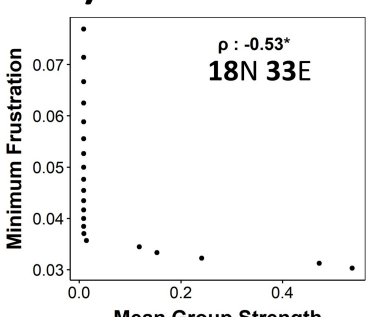

A iv)

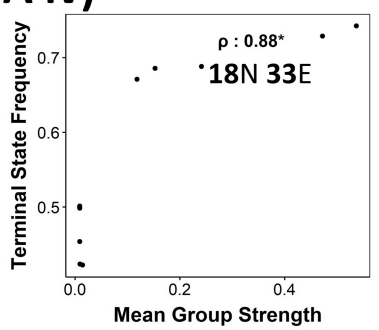

B

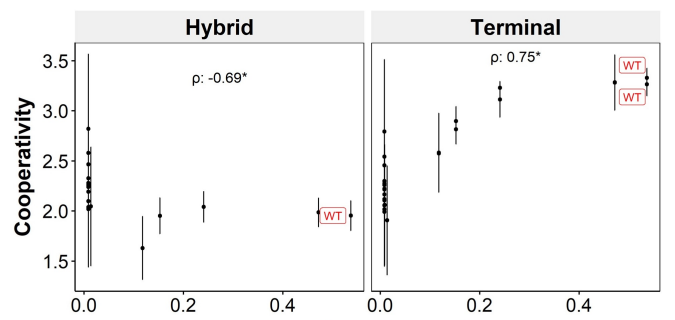

C

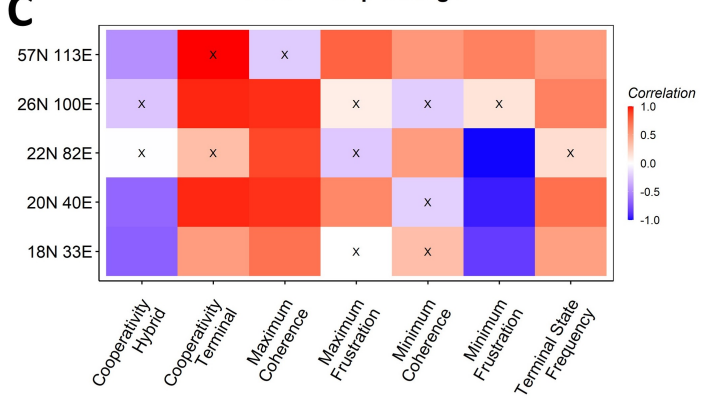

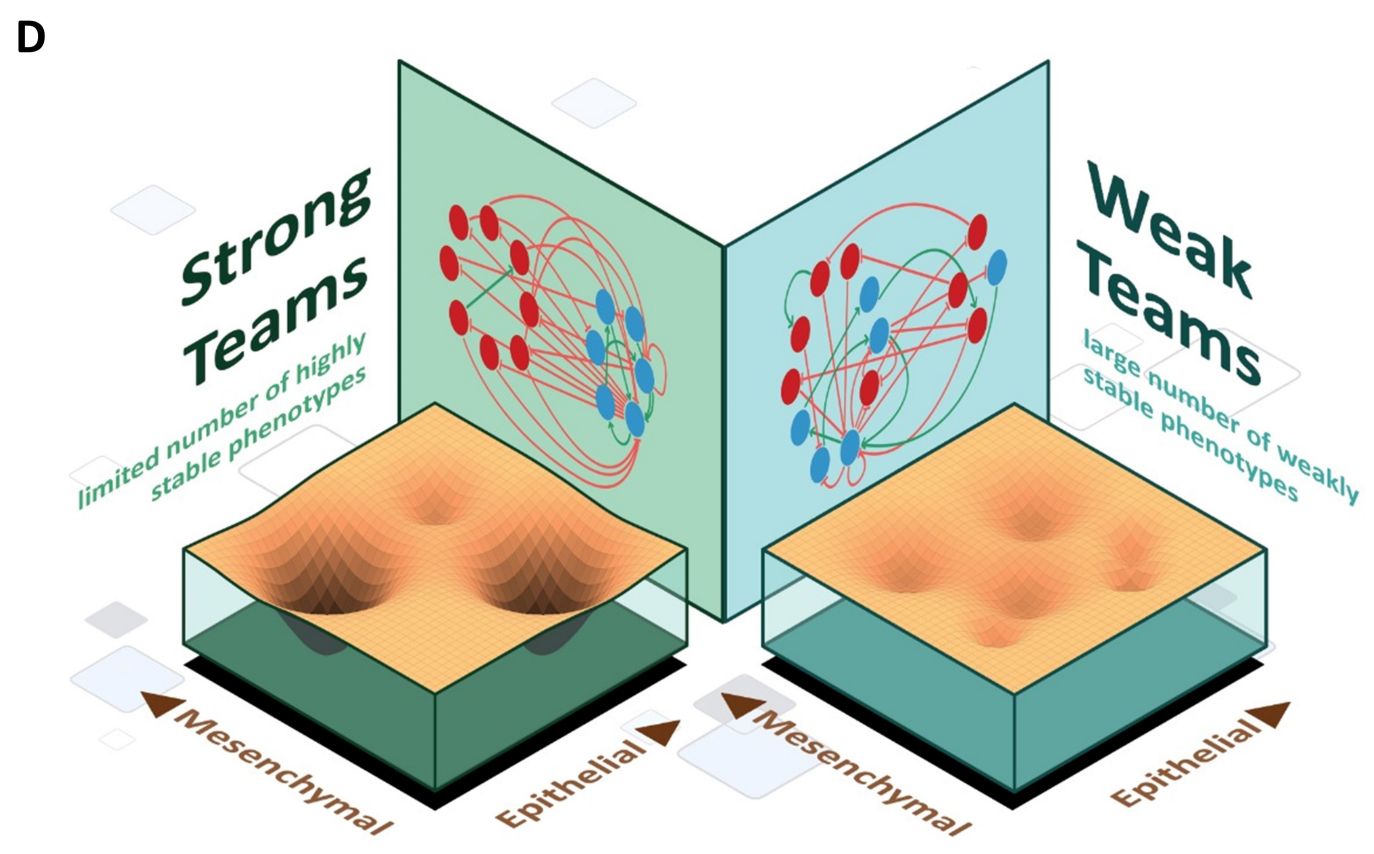

Figure 6: Reducing group strength leads to reduction in terminal phenotype stability (A) (i) Lineplot to demonstrate the reduction of group strength with each edge perturbed. Change in (ii) Minimum Frustration, (iii) Maximum Coherence and (iv) Terminal state frequency with increase in group strength. (B) Mean cooperativity for the perturbed networks against the mean group strength. (C) Correlation between group strength and stability metrics for the perturbed networks (edge deleted one at a time sequentially as shown for $18 \mathrm{~N} 33 \mathrm{E}$ network in panel A) obtained from all five EMP networks. (D) Schematic showing the effect of team structure on the EMP landscape

\section{Discussion}

Cellular decision processes often involve limited number of specific fates that they can switch among. In many cases, the underlying regulatory systems appear to be disproportionately more complex. In this study, we show how having teams of nodes is one way in which the networks 
achieve this property of having limited number of phenotypes despite their size and complexity.

Our first step was to identify teams of nodes in the network using unsupervised mechanisms, which we were able to accomplish by using hierarchical clustering on the influence matrix. Using the teams found in the influence matrix, we assigned a simple measure of the strength of teams (Gs). Gs was higher for the WT EMP networks as compared to the corresponding random networks generated by shuffling the edges between the nodes. One way to interpret this is the random networks are evolutionary alternatives that could have happened using the same set of nodes and edges, but the biological networks were selected to optimize for the strength of "teams". Similar analysis has been employed while understanding various other properties in previous studies. The fact that the WT network topologies do not have the absolute highest values for these properties is also a recurring theme: the reasons for which currently remain unclear (25).

The team structure we found is reminiscent of different kinds of modularity in large scale networks identified in previous studies. Particularly, two definitions of modularity are relevant. One is the existance of communities of nodes in the network that each perform a unique set of functions and have limited interactions with the other communities in the network(26). The two teams found in EMP networks do perform unique functions by supporting two mutually exclusive phenotypes (epithelial and mesenchymal), but there are extensive interactions between these teams also present, enabling a strong toggle switch between the teams. Another instance of modularity is the presence of strongly connected components $(27,28)$. This structure enables for an efficient transfer of information from the signal given to any of the nodes to all of the nodes, thereby showing coordinated expression of all components. While the teams do have the property of coordinated expression, connectedness is not a neccessary property of teams. The nodes of epithelial team in the $22 \mathrm{~N} 82 \mathrm{E}$ node network in our study do not have any interaction within themselves, but have coordinated expression only through the double negative loops they form with the nodes of the mesenchymal teams.

We defined the stability of the steady states emergent from these networks in 3 ways, covering the regimes of static (SSF and frustration) and dynamic (coherence) stability metrics. The first two metrics have been previously used to demonstrate the stability of EMP phenotypes, and our results echo these findings $(10,16)$, i.e., the epithelial and mesenchymal phenotypes show a higher SSF and lower frustration. Similarly, the WT networks have a lower frustration as compared to the random networks. These results resonate also with the team structure because an epithelial state perpetually had nodes of one team active and the other team inactive and vice-versa. This led to us 
classifying the epithelial and mesenchymal states as terminal states/phenotypes of the networks studied here.

The third metric, coherence, is an indirect measure of the attractor basin of a steady state, in that it measures the fraction of the neighbours of the steady state converging to it. There are multiple forms of this measure, defined depending upon the number of nodes being perturbed, and the measure being used for a state or a phenotype (Hamming distance to measure state similarity). Each of these forms revealed interesting insights into the stability characteristics of terminal phenotypes vs hybrid phenotypes. Cumulative coherence calculated over all possible single node perturbations revealed a bimodal distribution indicating terminal and hybrid phenotypes. Mean coherence for the perturbation of each node in the network revealed that the team structure is robust to perturbations in the signal nodes in the network. When we did multi-node perturbation, terminal phenotypes supported by teams retained their phenotypes longer than hybrid phenotypes. Interestingly, even for random networks where the group strength is low, the hybrid phenotypes of random networks did not necessarily show a better resilience than their terminal phenotypes.

One significant finding of our study is the identification of terminal and hybrid phenotypes purely based on the network topology, i.e., without having to simulate the network dynamics. While the biochemical composition of epithelial and mesenchymal phenotypes is relatively well defined across different cell types, the definition of hybrid phenotypes is quite context dependent $(29,28,30)$. One reason for this difference could be the non-uniformity in the gene lists used to identify these phenotypes from high-throughput data (31, 32). While our method of identification of hybrid phenotypes only considers the limited number of nodes available in the networks, the method itself is dependent on the way these nodes are interacting and not the nodes themselves. Hence, if we can infer the network from a set of genes in a cell type, using the teams approach, we can better define hybrid phenotypic signatures. Also, the hybrid phenotypes seen here are "metastable", similar to previous reports (10). Experimentally speaking, the frequency and stability of hybrid E/M phenotypes seems to be quite varied (32, 33, 29). This observation leads to question whether a third teamd comprising of factors driving a partial phenotype is required to stabilise hybrid E/M phenotypes, such as NP63 (34) and NRF2(35). Three teams of players have been proposed to give rise to three distinct phenotypes for instance in case of CD4+ T cell differentiation, where the three master regulators (and their corresponding team members) inhibit each other driving Th1, Th2 and Th17 phenotypes (36, 37). Another reason underlying higher stability of hybrid E/M phenotypes may be cell-cell communication and/or epigenetic regulations, both of 
which have not been included in our analysis.

While we specifically do not present any experimental evidence here, recent analysis based on pairwise correlations between master regulators of EMT/MET has revealed that MET inducers positively correlate with one another, but negatively with EMT inducers (38, 39). The existence of teams has also been seen in melanoma (40) and small cell lung cancer (12) where network topology and correlation matrices obtained from experimental data are quite consistent in decoding how nodes act together to decide certain phenotypes. Thus our results based on network topology can offer a possible mechanistic reason for why such teams are seen in correlation matrices for both in-vitro and in vivo data.

Here we see teams in terms of intracellular regulatory networks, but this framework of identifying composition of two (or more) groups acting together to reinforce each other in scenarios of competing outcome can be applied more broadly. For instance different cells in a microenvironment can form groups with pro tumor and anti-tumor inflence (41). How and when are these teams of cells formed remains to be identified. Also, how can these groups be broken to impact tumor progression needs to be investigated. Another conceptual question of interest is that how do teams bifurcate upon sequential instances of cellular differentiation (9). For instance two teams of nodes determine the decision between phenotype $A$ and phenotype $B$. Now when $B$ further differentiates to phenotypes $C$ and $D$, do new teams supporting $C$ and $D$ emerge or does the team $B$ break down into two sub teams? Identifying changes in regulatory networks that would be required to implement these rearrangements will be an interesting direction. Furthermore, answering whether the presence of teams necessary and sufficient to stabilize terminal phenotypes needs additional analysis.

Overall, our study highlights that despite their apparent complexity, there exist design principles hidden in network topology that constrain phenotypic space and increase phenotypic stability corresponding to large regulatory networks involved in cellular decision making (Fig 6D). Insights gained from such network topology and/or geometric approaches $(42,43)$ to studying gene regulatory networks can contribute to accurately identifying underlying landscape and modulating it for cellular reprogramming purposes. 


\section{Methods}

\subsection{Simulation Formalisms}

\subsubsection{RAndom Clrcuit PErturbaiton (RACIPE)}

RACIPE (15) is a tool that simulates transcriptional regulatory networks (TRNs) in a continuous manner. Given a TRN, it constructs a system of Ordinary Differential Equations representing the network. For a given node $T$ and a set of input nodes $P_{i}$ and $N_{j}$ that activate and inhibit $T$ respectively, the corresponding differential equation is given as equation 1 .

$$
\frac{d T}{d t}=G_{T} * \prod_{i} \frac{H^{S+}\left(P_{i}, P_{i T}^{0}, n_{P_{i}, T}, \lambda_{P_{i}, T}\right)}{\lambda_{P_{i}, T}} * \prod_{j} H^{S-}\left(N_{j}, N_{i T}^{0}, n_{N_{j}, T}, \lambda_{N_{j}, T}\right)-k_{T} * T
$$

Here, $T, P_{i}$ and $N_{j}$ represent the concentrations of the species. $G_{T}$ and $k_{T}$ denote the production and degradation rates, respectively. $P_{i T}^{0}$ is the threshold value of $P_{i}$ concentration at which the non-linearity in the dynamics of $T$ due to $P_{i}$ is seen. $n$ is termed as hill-coefficient and represents the extent of non-linearity in the regulation. $\lambda$ represents the fold change in the target node concentration upon over-expression of regulating node. Finally, the functions $H^{S+}$ and $H^{S-}$ are known as shifted hill functions (? ) and represent the regulation of the target node by the regulatory node (equation 2).

$$
H^{S+/-}\left(B, B_{A}^{0}, n_{B, A}, \lambda_{B, A}\right)=\frac{B_{A}^{0^{n_{B, A}}}}{B_{A}^{n_{B, A}}+B^{n_{B, A}}}+\lambda * \frac{B^{n_{B, A}}}{B_{A}^{n_{B, A}}+B^{n_{B, A}}}
$$

Note that, for high values of the regulatory node concentration, $H^{S+/-}$ approaches $\lambda$.

For the model generated in this way, RACIPE randomly samples parameter sets from a predefined set of parameter ranges estimated from BioNumbers (44). The default ranges used by RACIPE (15) are listed in Table 1.

At each parameter set, RACIPE integrates the model from multiple initial conditions and obtains steady states in the initial condition space. The output, hence, comprises of the collection of parameter sets and corresponding steady states obtained from the model. For the current analysis, we used a sample size of 10000 for parameter sets and 100 for initial conditions. The parameters were sampled via a uniform distribution and the ODE integration was carried out using Euler's method of numerical integration.

\subsubsection{Boolean simulations}

Boolean modelling is a logic-based, simple and discrete system for capturing the dynamics of biological networks. The framework describes each node of the network as a binary variable $(-1$ 
or 1) by considering a threshold value or quantity of the molecule that can elicit the necessary downstream function. In the framework used in this study (10) a state of a network is defined by a binary string of variables $s_{i}$, which gives information about which node is ON $\left(s_{i}=1\right)$ or OFF $\left(s_{i}=-1\right)$. The interactions between the nodes are represented using the non-symmetric adjacency matrix $\mathrm{J}$, where each element of the matrix, $J_{i j}$ is weight of the edge from $i^{t h}$ to $j^{t h}$ node of the network. All activations are given a weight 1 , and all inhibitions are given weight -1 . The simulations are carried out asynchronously (one randomly chosen node is updated at each iteration) based on experimental conditions, where the next iteration of the state is calculated using a majority rule given below:

$$
s_{i}(t+1)= \begin{cases}+1, \sum_{j} J_{i j} s_{j}(t)>0 & \\ -1, \sum_{j} J_{i j} s_{j}(t)<0 & \\ 0, & \text { otherwise }\end{cases}
$$

\subsubsection{Discretization of RACIPE output and calculating the state frequency}

For a given network with $i=[1, n]$ nodes, the steady state expression levels of the nodes were normalized about the mean and standard deviation across all parameter sets.

$$
Z_{i}=\frac{E_{i}-\overline{E_{i}}}{\sigma_{i}}
$$

Where, for the $i^{\text {th }}$ node, $E_{i}$ is the steady state expression level $\overline{E_{i}}$ is the combined mean and $\sigma_{i}$ is the combined standard deviation.

The z-scores are then classified based on whether they are negative or positive into 0 (low) and 1 (high) expression levels respectively. Each steady state of the network is thus labelled with a string of 1's and 0's, discretizing the continuous steady state levels. We then calculate the total frequency of each discrete state by counting the occurrence in all the parameter sets. For parameter sets with $n$ steady states, the count of each steady state is taken as $1 / n$, invoking the assumption that all the states are equally stable.

\subsubsection{Quantitative convergence}

To estimate the optimal sample size of parameter sets for RACIPE and that of initial conditions for Boolean models, all networks were simulated at different sample sizes in triplicates and the mean an variance of the steady state frequency distribution was calculated. 10000 was estimated as the ideal sample sizes for both methods as it was the smallest sample size for which the variance in steady state frequencies was minimum and the mean of the same was consistently similar to that of higher sample sizes. 


\subsection{Figure 1}

\subsubsection{Network Visualization}

Cytoscape 3.9.0 (45) was used to visualize the networks studied. Edges were colored based on the sign (inhibiting and activating) and nodes were colored based on their nature (epithelial, mesenchymal and peripheral).

\subsubsection{Influence Matrix, group strength and state strength}

The Influence Matrix, as the name suggests, is a matrix where each element at $(\mathrm{i}, \mathrm{j})$ position records the influence of ith node on the jth node in the network, mediated through one or more serially connected edges that form a path from ith node to the jth node in the network. Path length is defined as the length of such paths being considered for the calculation of influence. As a result, for a path length of 1 , the influence matrix is equivalent to the adjacency matrix J. For a path length of $\mathrm{I}$, the influence is calculated as $J^{l}$. In the same manner, the influence is calculated for all path lengths till $l_{\max }=10$ edges. Finally, the influence matrix for a path length of $l_{m} a x$ is calculated by merging the matrices $J^{l}$ as given in Equation 5 (12).

$$
\text { Inf } l_{\max }=\frac{\sum_{l=1}^{l_{\max }} \frac{J^{l}}{J_{l_{\max }}^{l}}}{l_{\max }}
$$

Where $J_{\max }$ denotes the magnitude of the greatest possible interaction of a given network architecture, derived by putting all non-zero entries of the adjacency matrix to 1 , and is thus utilised as the normalising factor. The division $J^{l} /\left(J_{\text {max }}^{l}\right)$ is element-wise. The division with $l_{\text {max }}$ ensures that the elements of $I n f_{l_{\max }}$ are constrained between -1 and 1.

\subsubsection{Random Network Generation}

Generation of random networks is an important technique that enables us to analyse the similarities and/or differences between biological networks and networks that do not occur in nature (essentially 'random'). To create random networks, we start with a biological network and randomly rearrange the 'Target' nodes, which essentially leads to a random rearrangement of the edges. This exercise was repeated multiple times $(k=10)$ to create random networks. This scheme conserves node degree, activatory and inhibitory contacts, and the number of nodes activated and inhibited. 


\subsubsection{Distance between influence and interaction matrices}

Distance between influence and correlation matrices was calculated using the following formula:

$$
d=\sum_{i=1}^{N} \sum_{j=1}^{N} \frac{\mid \text { Cor }_{i j}-\operatorname{Infl} l_{i j} \mid}{2 N^{2}}
$$

Where $C o r$ is the correlation matrix and $I n f l$ is the influence matrix.

\subsection{Figure 3}

\subsubsection{Coherence}

Coherence is calculated by perturbing the node expression levels in a state. 'Perturbation' in the context of Boolean networks corresponds to essentially flipping or reversing a state. For instance, if level of $S_{i}$ is ON (1), then perturbing it entails switching it to OFF $(-1)$. The general form is given below:

$$
s_{i}^{\text {pert }}=\left\{\begin{array}{l}
1, s_{i}=-1 \\
-1, s_{i}=1
\end{array}\right.
$$

Where $n_{i}^{\text {pert }}$ is the perturbed node. Node perturbations were done for every node in every steady state one at a time. For a steady state $S$, coherence is defined as the probability that simulation followed by a single node perturbation of that state would result in the original steady state. To calculate coherence of a steady state, we perturb the state at one node at a time simulate the network with the perturbed state as the initial condition. For each simulation, we assign a score of 1 if it the original state is achieved, and 0 if it is not. We repeat these simulations for each node in the network for $\mathrm{K}=100$ iterations, and define coherence as the average of the assigned scores over all simulations as follows:

$$
\text { Coherence }_{S}=\frac{\sum_{k=1}^{N} \sum_{i=1}^{N}\left\{\begin{array}{l}
1, S^{\text {pert }}=S \\
0, S^{\text {pert }} \neq S
\end{array}\right.}{N * K}
$$

Where $S_{i}^{\text {pert }}$ is the steady state obtained after simulation of the perturbed steady-state and $\mathrm{N}$ is the number of nodes in the network.

\subsubsection{EMT Score}

EMT score is a metric devised to quantify how epithelial or how mesenchymal a given state is. It is on a scale of -1 to 1 , where -1 is fully epithelial and 1 is fully mesenchymal. For a state $S$, the EMT score is calculated as follows:

$$
\text { EMTscore }_{S}=\frac{\sum S_{E}}{n_{E}}-\frac{\sum S_{M}}{n_{M}}
$$


Where $S_{E}$ is the state of the core epithelial nodes and $S_{M}$ is the states of core mesenchymal nodes in the 0,1 representation, and $n_{E}$ and $n_{M}$ are the number of core epithelial and the core mesenchymal nodes in the network respectively. The core nodes refer to the nodes of the network that are neither signal nor output nodes (i.e., the corresponding row and column in the adjacency matrix have atleast one non-empty element).

\subsubsection{State Strength}

Strength of a state $S$ is calculated using the following formula:

$$
\text { Strength }_{S}=\sum_{i, j=1}^{N} \operatorname{Infl}_{i j} S_{i} S_{j}
$$

\subsection{Figure 5}

\subsubsection{Multinode perturbation}

We performed experiments to characterize the stability of terminal and hybrid steady states over ' $n$ ' node perturbations, ' $n$ ' ranging from 1 to $\mathrm{N}$ (total number of nodes in a given network). Essentially, this means perturbing ' $n$ ' nodes at a time for each steady state, instead of perturbing just one node as seen for coherence calculations. For simplicity, the number of such perturbations for a particular value of 'n' was decided by the following rule

$$
\text { Numberofperturbations }=\min \left(100,\left(\begin{array}{l}
N \\
n
\end{array}\right)\right)
$$

The perturbed steady states were then simulated using Boolean formalism for 1000-time steps, with 10 repeats to accomodate the fact that asynchronous boolean simulations can allow a single initial condition to converge to multiple steady states. The final state obtained is compared with the original steady state by employing measures such as coherence and Hamming distance. The latter entails comparing these two states by considering the number of bit positions in which the two bits are different. The EMT score is also calculated for the final state obtained. We repeat these simulations for each ' $n$ ' number of perturbations in the network for $K=100$ iterations, and average out the final Coherence, Hamming and EMT Score values for each original steady state.

\subsection{Statistical tests}

All correlation analysis was done using Spearman correlation method using 'cor.test' function in $R$ 4.0.2. The corresponding statistical significance values are represented by '*'s, to be translated as: *: $p<0.05$. 


\section{Author Contributions}

MKJ designed and supervised research; $K H, V U, A B$ and $A G$ conducted research and analyzed the data; all authors discussed results and participated in the preparation of the manuscript.

\section{Conflict of Interest}

The authors declare no competing financial or non-financial interests.

\section{Data and Code Availability}

The simulation data, and codes used for the analysis and figure generation are available on the github page: https://github.com/askhari139/TeamsProject.

\section{Acknowledgements}

This work was supported by Ramanujan Fellowship awarded to MKJ by Science and Engineering Research Board (SERB), Department of Science and Technology (DST), Government of India (SB/S2/RJN-049/2018), by Infosys Young Investigator award to MKJ supported by Infosys Foundation, Bangalore and by Prime Minister's Research Fellowship awarded to KH. Atchuta Srinivas Duddu is acknowledged for artwork (Fig 3A, Fig 6D) 


\section{References}

[1] Balázsi, G., Oudenaarden, A. V. \& Collins, J. J. Cellular decision making and biological noise: from microbes to mammals. Cell 144, 910-925 (2011).

[2] Guantes, R. \& Poyatos, J. F. Multistable decision switches for flexible control of epigenetic differentiation. PLOS Computational Biology 4, e1000235 (2008).

[3] Prochazka, L., Benenson, Y. \& Zandstra, P. W. Synthetic gene circuits and cellular decisionmaking in human pluripotent stem cells. Current Opinion in Systems Biology 5, 93-103 (2017).

[4] Agozzino, L., Balázsi, G., Wang, J. \& Dill, K. A. How do cells adapt? stories told in landscapes. Annual review of chemical and biomolecular engineering 11, 155 (2020).

[5] Ozbudak, E. M., Thattal, M., Lim, H. H., Shraiman, B. I. \& Oudenaarden, A. V. Multistability in the lactose utilization network of escherichia coli. Nature 2004 427:6976 427, 737-740 (2004).

[6] Enane, F. O., Saunthararajah, Y. \& Korc, M. Differentiation therapy and the mechanisms that terminate cancer cell proliferation without harming normal cells. Cell Death Disease 2018 9:9 9, 1-15 (2018).

[7] McKimpson, W. M. \& Accili, D. Reprogramming cells to make insulin. Journal of the Endocrine Society 3, 1214 (2019).

[8] Newman, S. A. Cell differentiation: What have we learned in 50 years? Journal of theoretical biology 485 (2020).

[9] Zhou, J. X. \& Huang, S. Understanding gene circuits at cell-fate branch points for rational cell reprogramming. Trends in Genetics 27, 55-62 (2011).

[10] Font-Clos, F., Zapperi, S. \& Porta, C. A. M. L. Topography of epithelial-mesenchymal plasticity. Proceedings of the National Academy of Sciences 115, 5902-5907 (2018).

[11] Gates, A. J., Correia, R. B., Wang, X. \& Rocha, L. M. The effective graph reveals redundancy, canalization, and control pathways in biochemical regulation and signaling. Proceedings of the National Academy of Sciences of the United States of America 118 (2021). 
[12] Chauhan, L., Ram, U., Hari, K. \& Jolly, M. K. Topological signatures in regulatory network enable phenotypic heterogeneity in small cell lung cancer. eLife 10 (2021).

[13] Silveira, D. A. \& Mombach, J. C. M. Dynamics of the feedback loops required for the phenotypic stabilization in the epithelial-mesenchymal transition. The FEBS Journal 287, 578-588 (2019).

[14] Silveira, D. A., Gupta, S. \& Mombach, J. C. M. Systems biology approach suggests new mirnas as phenotypic stability factors in the epithelial-mesenchymal transition. Journal of the Royal Society Interface 17 (2020).

[15] Huang, B. et al. Interrogating the topological robustness of gene regulatory circuits by randomization. PLOS Computational Biology 13, e1005456 (2017).

[16] Tripathi, S., Kessler, D. A. \& Levine, H. Biological networks regulating cell fate choice are minimally frustrated. Physical Review Letters 125, 088101 (2020).

[17] Tripathi, S., Levine, H. \& Jolly, M. K. The physics of cellular decision making during epithelialmesenchymal transition. https://doi.org/10.1146/annurev-biophys-121219-081557 49, 1-18 (2020).

[18] Deshmukh, A. P. et al. Identification of emt signaling cross-talk and gene regulatory networks by single-cell rna sequencing. Proceedings of the National Academy of Sciences of the United States of America 118, e2102050118 (2021).

[19] Brabletz, S. \& Brabletz, T. The ZEB/miR-200 feedback loop-a motor of cellular plasticity in development and cancer? EMBO reports 11, 670-677 (2010).

[20] Plygawko, A. T., Kan, S. \& Campbell, K. Epithelial-mesenchymal plasticity: emerging parallels between tissue morphogenesis and cancer metastasis. Philosophical Transactions of the Royal Society B 375, 20200087 (2020).

[21] Ellison, A. M. EFFECT OF SEED DIMORPHISM ON THE DENSITY-DEPENDENT DYNAMICS OF EXPERIMENTAL POPULATIONS OF ATRIPLEX TRIANGULARIS (CHENOPODIACEAE). American Journal of Botany 74, 1280-1288 (1987).

[22] Willadsen, K. \& Wiles, J. Robustness and state-space structure of boolean gene regulatory models. Journal of theoretical biology 249, 749-765 (2007). 
[23] George, J. T., Jolly, M. K., Xu, S., Somarelli, J. A. \& Levine, H. Survival outcomes in cancer patients predicted by a partial emt gene expression scoring metric. Cancer research 77, 6415-6428 (2017).

[24] Nieto, M. $\square$. , Huang, R. Y., Jackson, R. A. \& Thiery, J. P. Emt: 2016. Cell 166, 21-45 (2016).

[25] Hebbar, A., Moger, A., Hari, K. \& Jolly, M. K. Interplay of positive and negative feedback loops governs robustness in multistable biological networks. bioRxiv 2021.10.08.463488 (2021).

[26] Wang, R.-S. \& Albert, R. Effects of community structure on the dynamics of random threshold networks. Physical Review E 87 (2013).

[27] Zañudo, J. G. T. \& Albert, R. An effective network reduction approach to find the dynamical repertoire of discrete dynamic networks. Chaos: An Interdisciplinary Journal of Nonlinear Science 23, 025111 (2013).

[28] Steinway, S. N. et al. Combinatorial interventions inhibit TGF-driven epithelial-tomesenchymal transition and support hybrid cellular phenotypes. npj Systems Biology and Applications 1 (2015).

[29] Jolly, M. K. et al. Stability of the hybrid epithelial/mesenchymal phenotype. Oncotarget 7, 27067-27084 (2016).

[30] Watanabe, K., Panchy, N., Noguchi, S., Suzuki, H. \& Hong, T. Combinatorial perturbation analysis reveals divergent regulations of mesenchymal genes during epithelial-tomesenchymal transition. npj Systems Biology and Applications 5 (2019).

[31] Puram, S. V. et al. Single-cell transcriptomic analysis of primary and metastatic tumor ecosystems in head and neck cancer. Cell 171, 1611-1624.e24 (2017).

[32] Pastushenko, I. et al. Identification of the tumour transition states occurring during EMT. Nature 556, 463-468 (2018).

[33] Ruscetti, M. et al. HDAC inhibition impedes epithelial-mesenchymal plasticity and suppresses metastatic, castration-resistant prostate cancer. Oncogene 35, 3781-3795 (2015).

[34] Dang, T. T., Esparza, M. A., Maine, E. A., Westcott, J. M. \& Pearson, G. W. \{upDeltanp63 promotes breast cancer cell motility through the selective activation of components of the epithelial-to-mesenchymal transition program. Cancer Research 75, 3925-3935 (2015). 
[35] Bocci, F. et al. NRF2 activates a partial epithelial-mesenchymal transition and is maximally present in a hybrid epithelial/mesenchymal phenotype. Integrative Biology 11, 251-263 (2019).

[36] Zhu, J., Yamane, H. \& Paul, W. E. Differentiation of effector CD4 t cell populations. Annual Review of Immunology 28, 445-489 (2010).

[37] Duddu, A. S., Sahoo, S., Hati, S., Jhunjhunwala, S. \& Jolly, M. K. Multi-stability in cellular differentiation enabled by a network of three mutually repressing master regulators. Journal of The Royal Society Interface 17, 20200631 (2020).

[38] Chakraborty, P. et al. Analysis of immune subtypes across the epithelial-mesenchymal plasticity spectrum. Computational and Structural Biotechnology Journal 19, 3842-3851 (2021).

[39] Jia, W. et al. Epigenetic feedback and stochastic partitioning during cell division can drive resistance to EMT. Oncotarget 11, 2611-2624 (2020).

[40] Pillai, M. \& Jolly, M. K. Systems-level network modeling deciphers the master regulators of phenotypic plasticity and heterogeneity in melanoma. iScience 24, 103111 (2021).

[41] Capp, J.-P. et al. Group phenotypic composition in cancer. eLife 10 (2021).

[42] Sáez, M. et al. Statistically derived geometrical landscapes capture principles of decisionmaking dynamics during cell fate transitions. Cell Systems (2021).

[43] Rand, D. A., Raju, A., Sáez, M., Corson, F. \& Siggia, E. D. Geometry of gene regulatory dynamics. Proceedings of the National Academy of Sciences 118, e2109729118 (2021).

[44] Milo, R., Jorgensen, P., Moran, U., Weber, G. \& Springer, M. BioNumbers-the database of key numbers in molecular and cell biology. Nucleic Acids Research 38, D750-D753 (2009).

[45] Shannon, P. et al. Cytoscape: A software environment for integrated models of biomolecular interaction networks. Genome Research 13, 2498-2504 (2003). 
bioRxiv preprint dol: https://doi.org/10.1101/2021.12.12.472090; this version posted December 13, 2021. The copyright holder for this preprint (which was not certified by peer review) is the author/funder, who has granted bioRxiv a license to display the preprint in perpetuity. It is made available under aCC-BY-NC-ND 4.0 International license.

A

$18 \mathrm{~N} 33 \mathrm{E}$

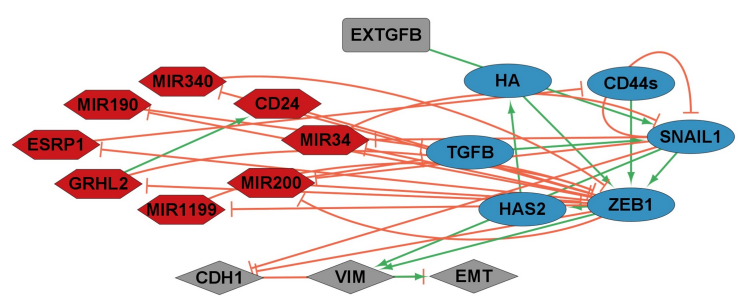

C $\quad 26 \mathrm{~N} 100 \mathrm{E}$

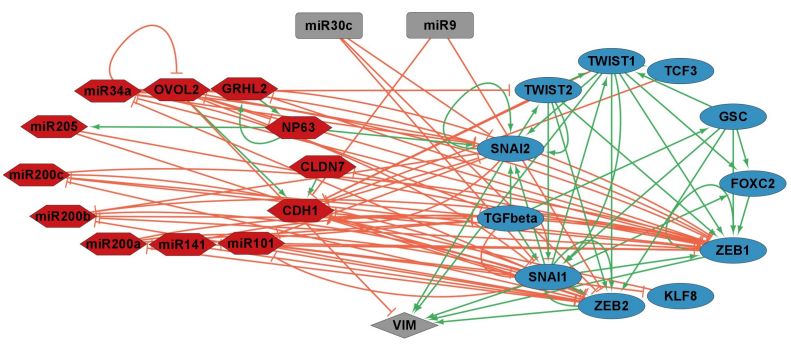

B

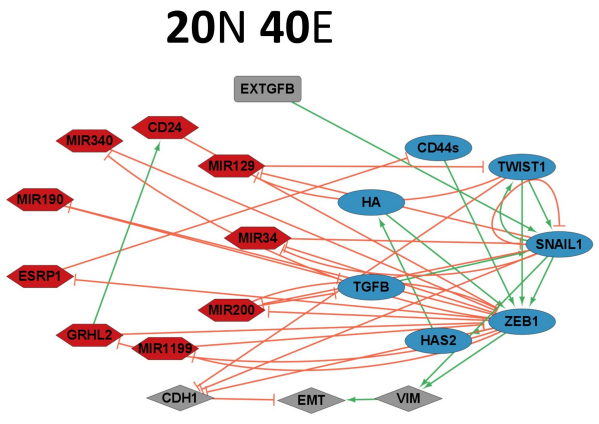

D

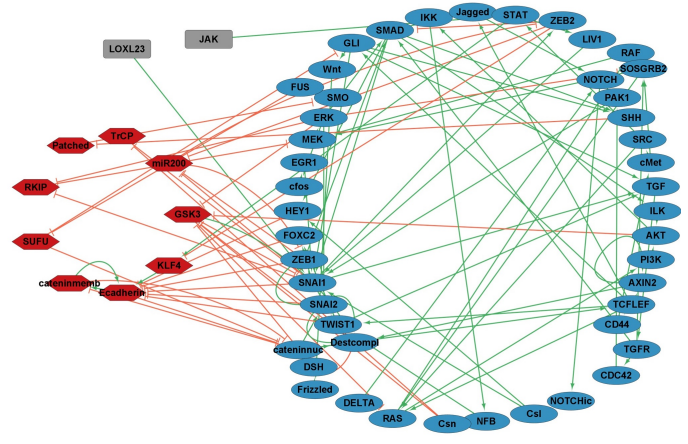

Fig S1. EMP networks considered for simulation of sizes (A) 18N 33E, (B) 20N 40E, (C) $26 \mathrm{~N} 100 \mathrm{E}$ and (D) $57 \mathrm{~N} 113 \mathrm{E}$. The nodes colored as : red - epithelial, blue- mesenchymal, grey - peripheral nodes

36 
bioRxiv preprint doi: https://doi.org/10.1101/2021.12.12.472090; this version posted December 13, 2021. The copyright holder for this preprint (which was not certified by peer review) is the author/funder, who has granted bioRxiv a license to display the preprint in perpetuity. It is made available under aCC-BY-NC-ND 4.0 International license.
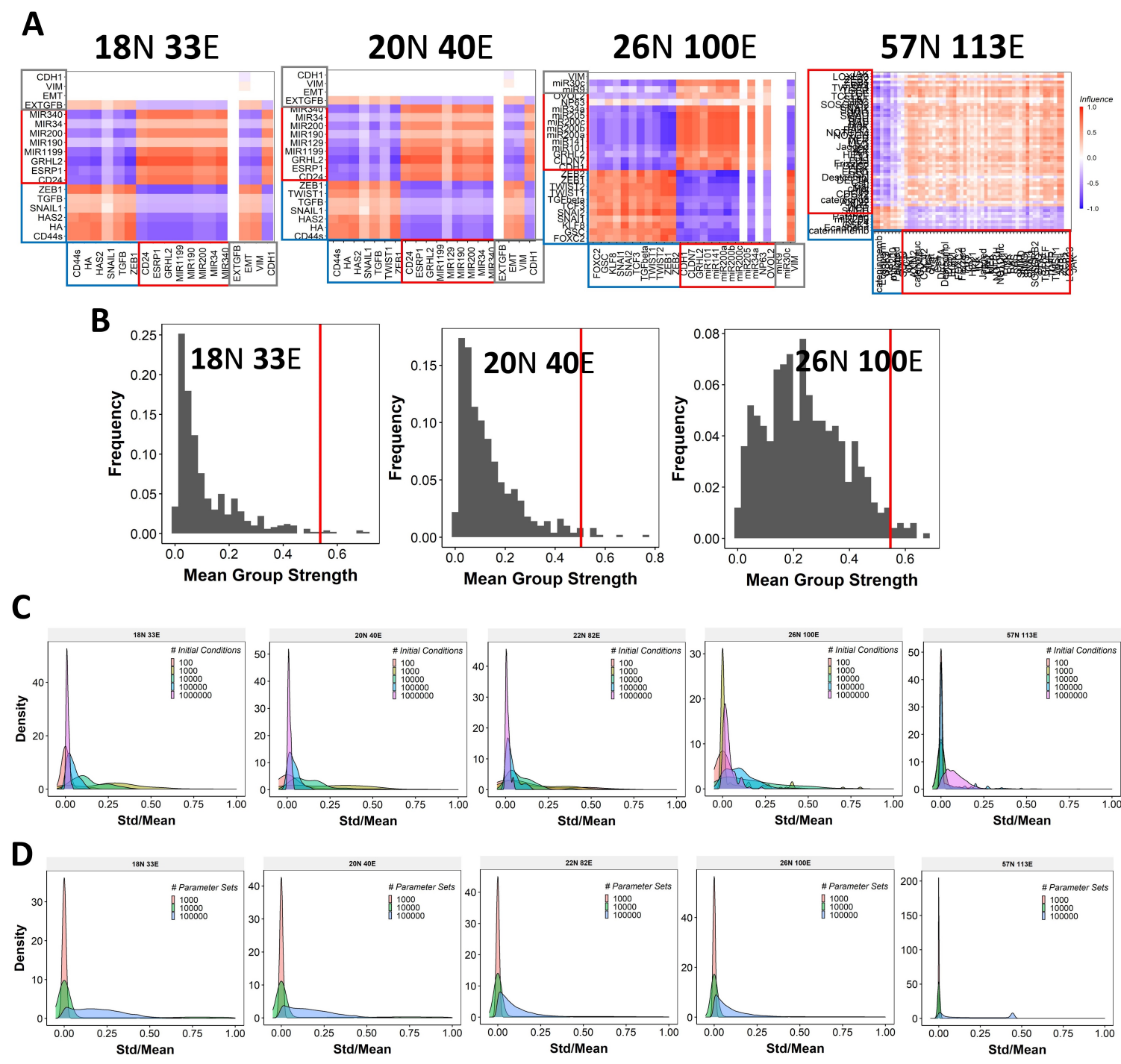

E
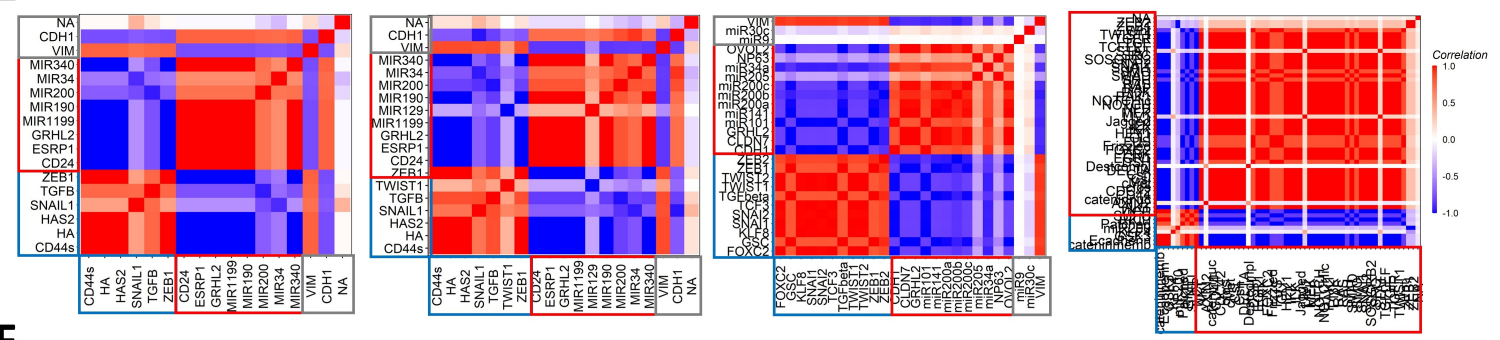

F
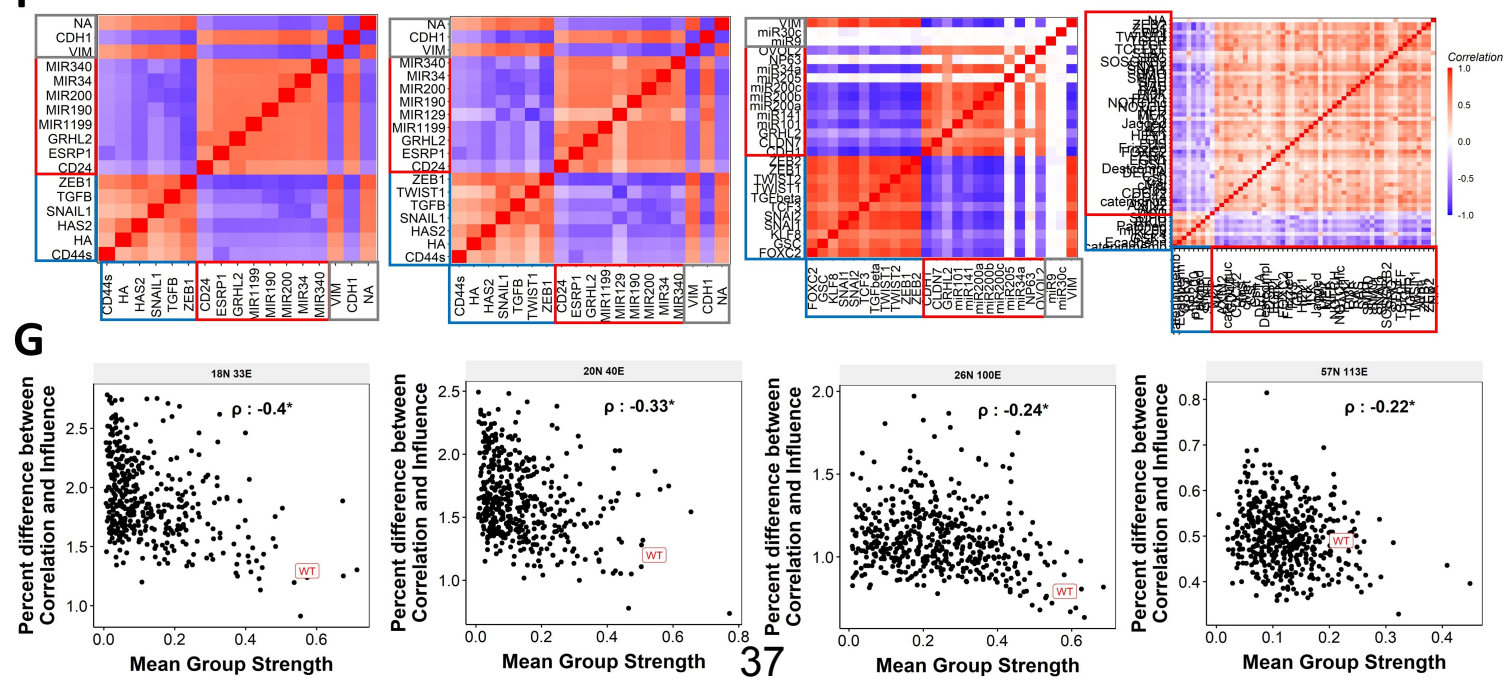
Fig S2.(A) Influence matrices for the EMP networks of size (i) $18 \mathrm{~N} 33 \mathrm{E}$, (ii) $20 \mathrm{~N} 40 \mathrm{E}$ (iii) $26 \mathrm{~N}$ $100 \mathrm{E}$ and (iv) $57 \mathrm{~N} 113 \mathrm{E}$. (B) Distribution of the mean group strength (Gs) for EMP networks (i) $18 \mathrm{~N} 33 \mathrm{E}$, (ii) $20 \mathrm{~N} 40 \mathrm{E}$ and (ii) $26 \mathrm{~N} 100 \mathrm{E}$. (C) Distribution of the ratio of standard deviation and mean obtained for the steady state distributions of Boolean simulations with multiple values of the number of initial conditions (color) for EMP networks of sizes (i) $18 \mathrm{~N} 33 \mathrm{E}$, (ii) $20 \mathrm{~N} 40 \mathrm{E}$, (iii) $22 \mathrm{~N}$ $82 \mathrm{E}$, (iv) $26 \mathrm{~N} 100 \mathrm{E}$, (v) $57 \mathrm{~N} 113 \mathrm{E}$. (D) Same as C but for RACIPE (E) Correlation matrices for RACIPE expression levels of EMP networks, in increasing order of number of nodes except for $22 \mathrm{~N}$ 82E network. (F) Same as $\mathrm{G}$ but for Boolean. (G) Similar to Fig 1F, for networks other than $22 \mathrm{~N} 82 \mathrm{E}$.

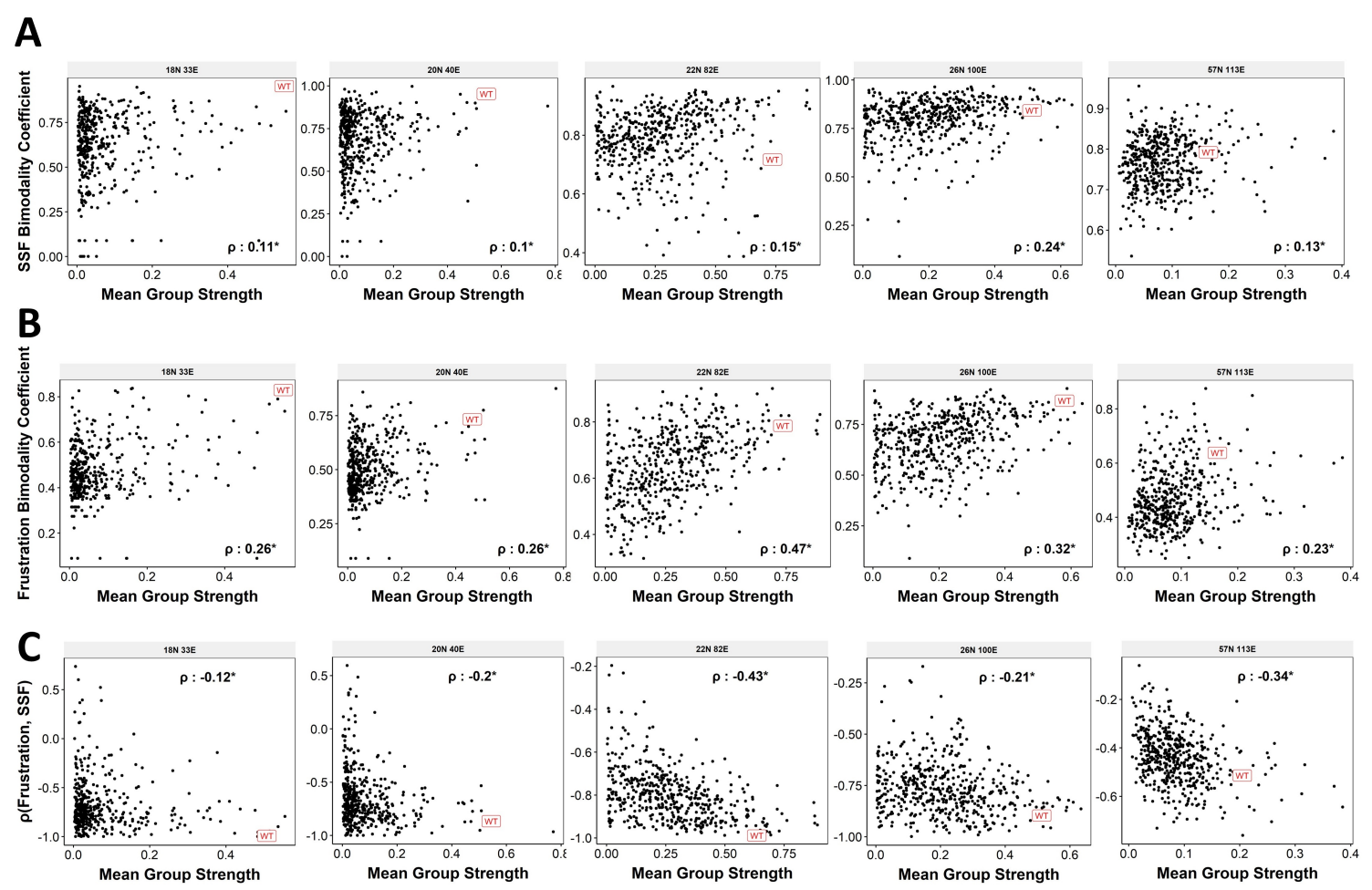

D
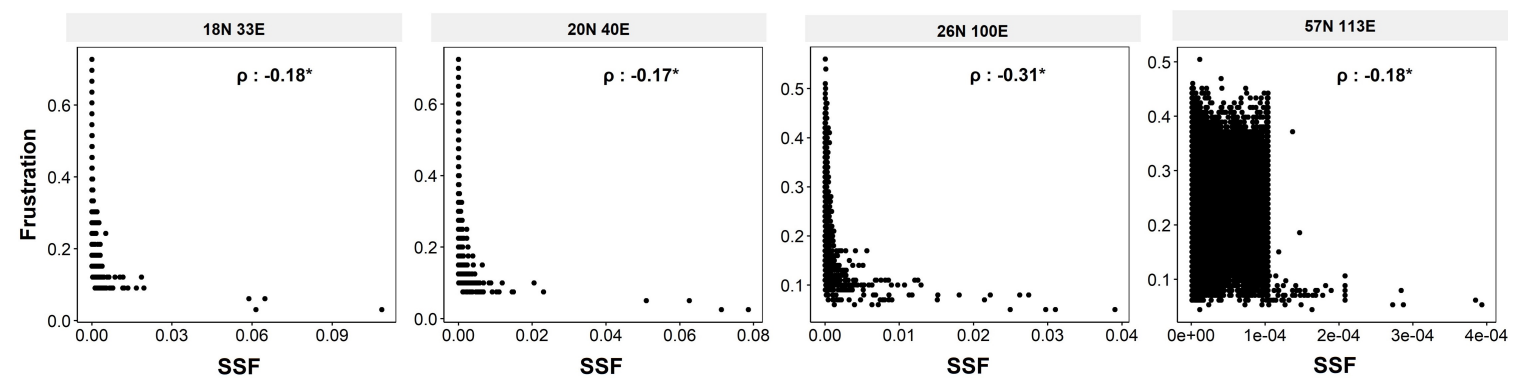

Fig S3.(A) Bimodality coefficient of steady state frequency against the group strength. Each dot is a random network. The WT EMP network is labelled in red. (B) Same as A but for bimodality coefficient for Frustration (C) Same as A but for correlation between Frustration and SSF. (D) Frustration vs SSF scatterplots for RACIPE simulations. 
bioRxiv preprint doi: https://doi.org/10.1101/2021.12.12.472090; this version posted December 13,2021 . The copyright holder for this preprint (which was not certified by peer review) is the author/funder, who has granted bioRxiv a license to display the preprint in perpetuity. It is made available under aCC-BY-NC-ND 4.0 International license.
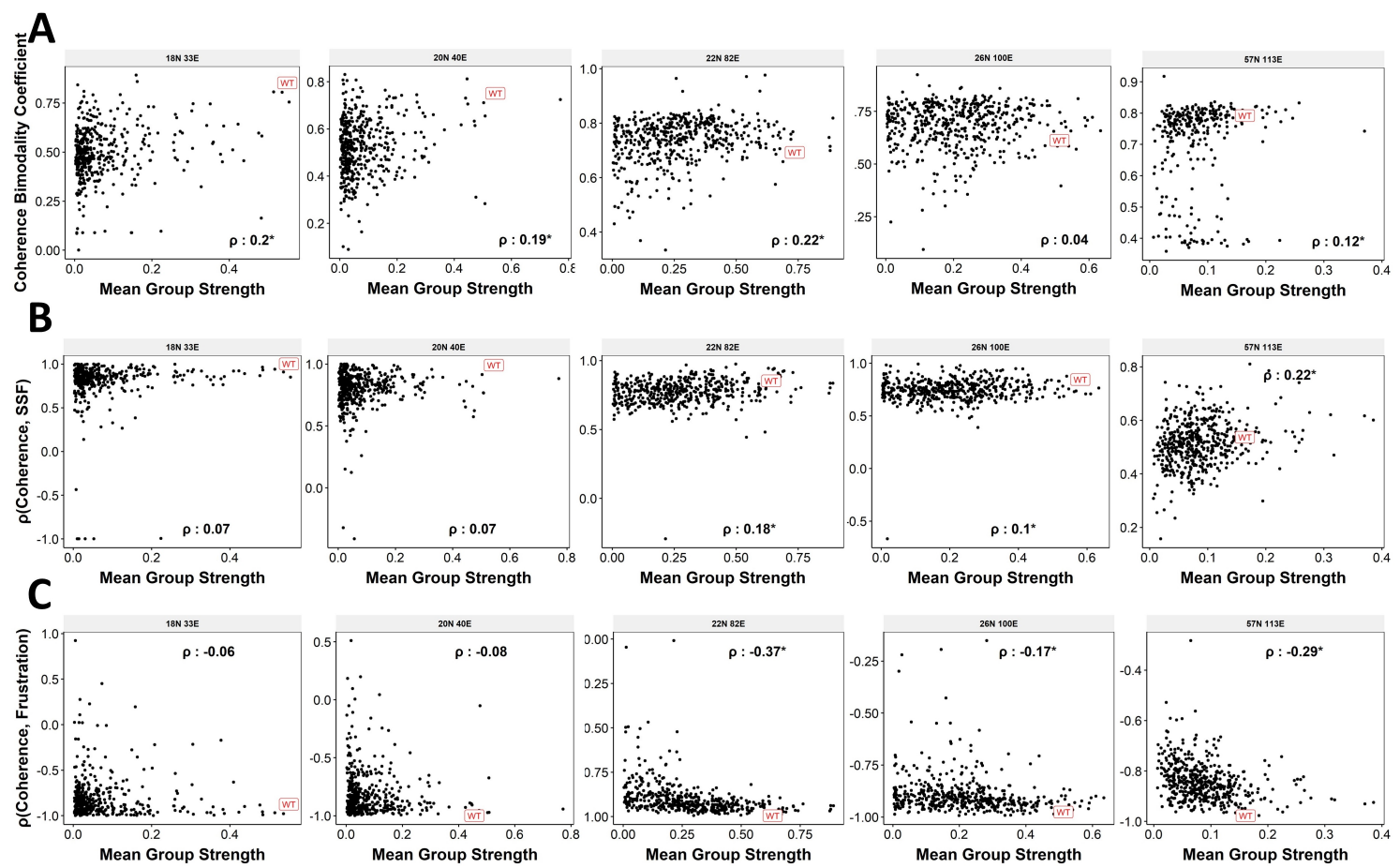

Fig S4.(A) Bimodality coefficient of coherence against the group strength. Each dot is a random network. The WT EMP networks are labelled in red. (B) Same as A, but for correlation coefficient of coherence and SSF. (C) Same as A, but for correlation coefficient of coherence and frustration. 
bioRxiv preprint doi: https://doi.org/10.1101/2021.12.12.472090; this version posted December 13,2021. The copyright holder for this preprint (which was not certified by peer review) is the author/funder, who has granted bioRxiv a license to display the preprint in perpetuity. It is made available under aCC-BY-NC-ND 4.0 International license.

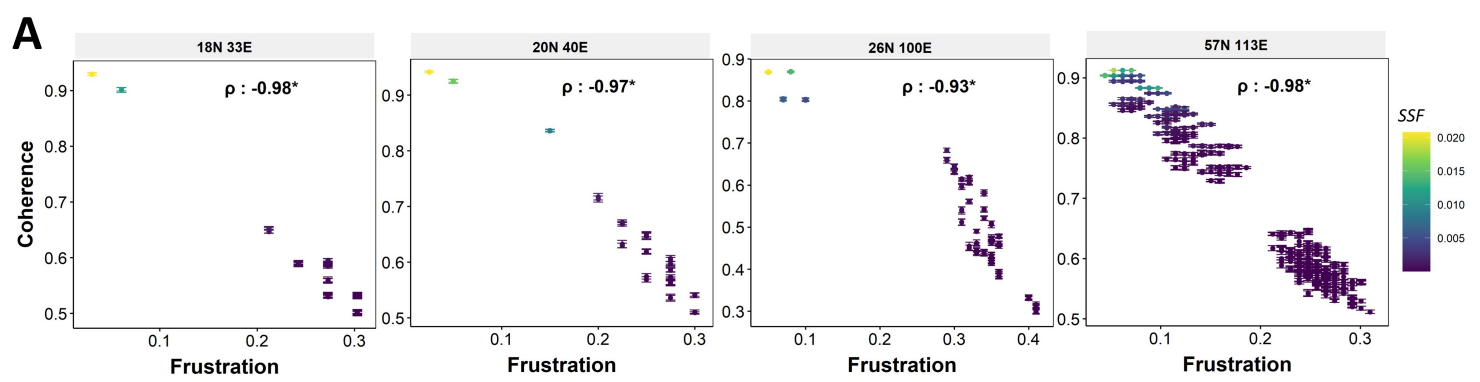

B
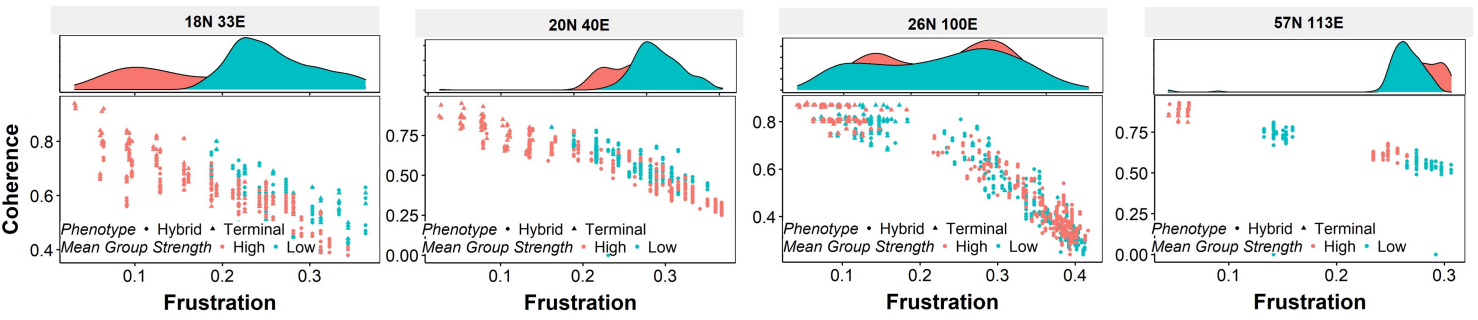

C
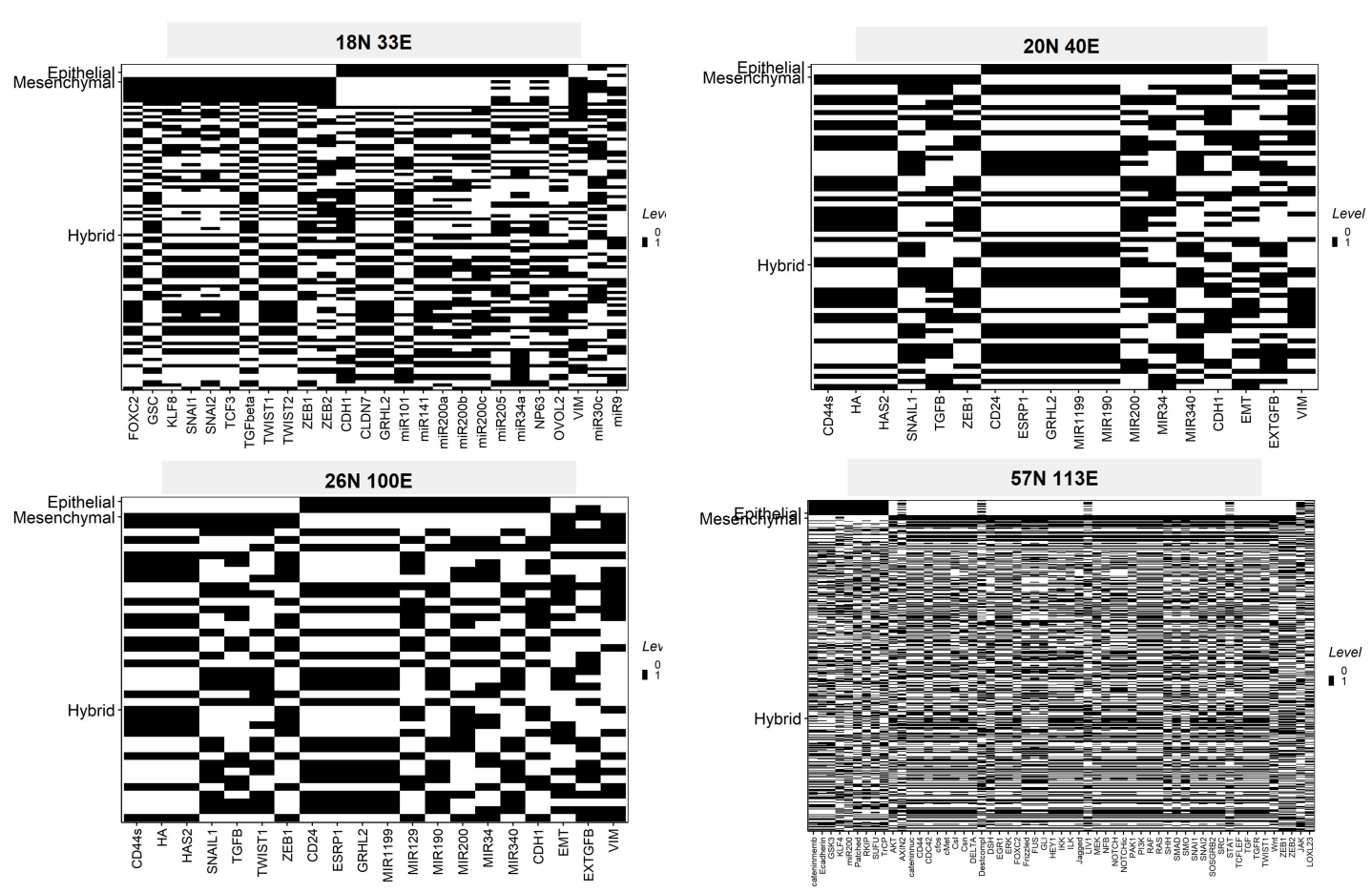

Fig S5.(A) Coherence, Frustration and SSF of the steady states of WT EMP networks. (B) Same as Fig 4Aii, for networks of sizes not shown in main. (C) Steady state composition matrices of EMP networks. 
bioRxiv preprint doi: https://doi.org/10.1101/2021.12.12.472090; this version posted December 13, 2021. The copyright holder for this preprint (which was not certified by peer review) is the author/funder, who has granted bioRxiv a license to display the preprint in perpetuity. It is made available under aCC-BY-NC-ND 4.0 International license.

A
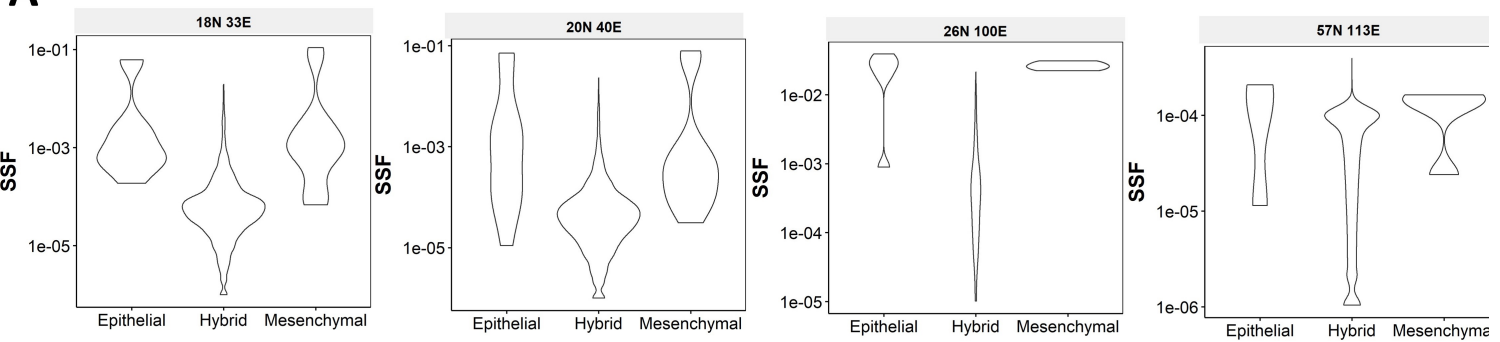

B
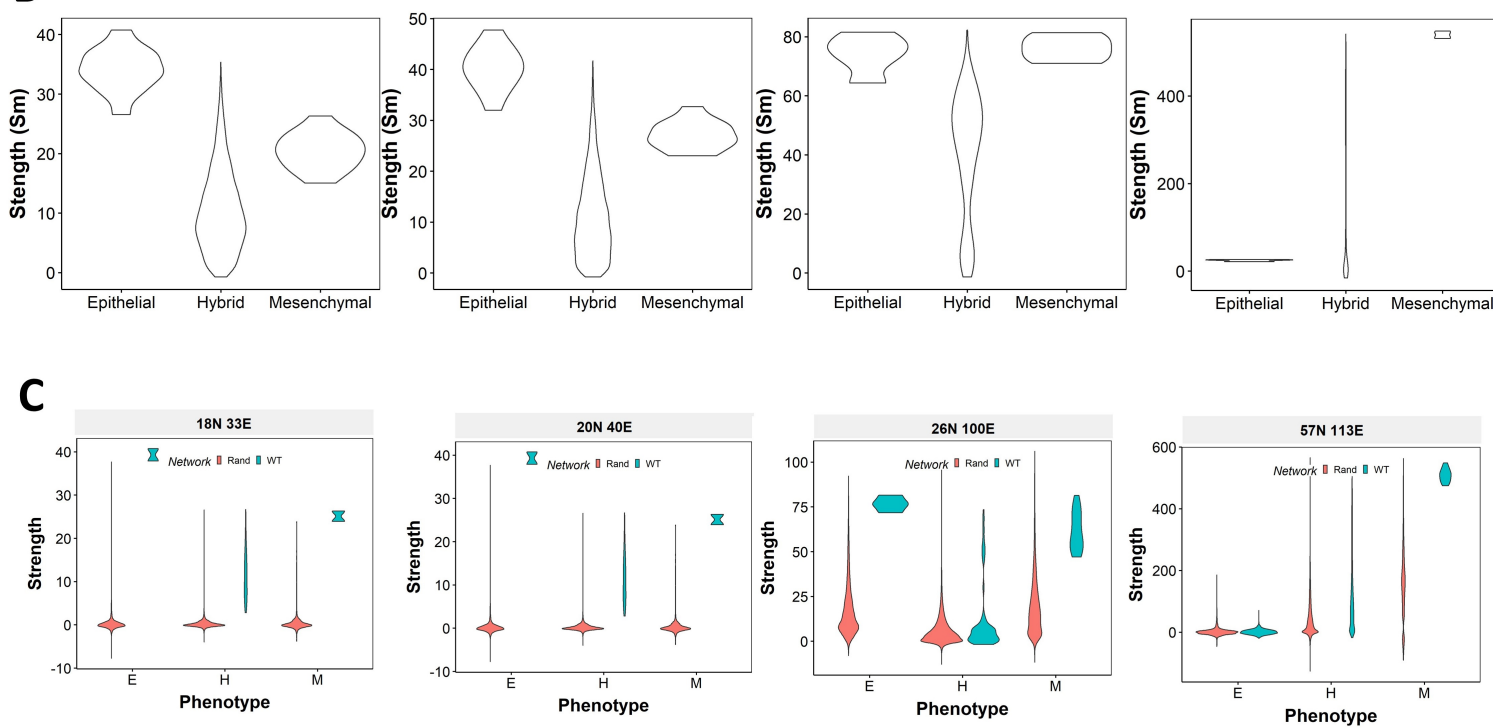

Fig S6.(A) SSF distribution for Epithelial, Mesenchymal and Hybrid steady states of EMP networks obtained from RACIPE. (B) Distribution of strength of state for RACIPE steady states of EMP networks (C) Comparison of state strength distribution for random and WT EMP networks. 
bioRxiv preprint doi: https://doi.org/10.1101/2021.12.12.472090; this version posted December 13, 2021. The copyright holder for this preprint (which was not certified by peer review) is the author/funder, who has granted bioRxiv a license to display the preprint in perpetuity. It is made available under aCC-BY-NC-ND 4.0 International license.
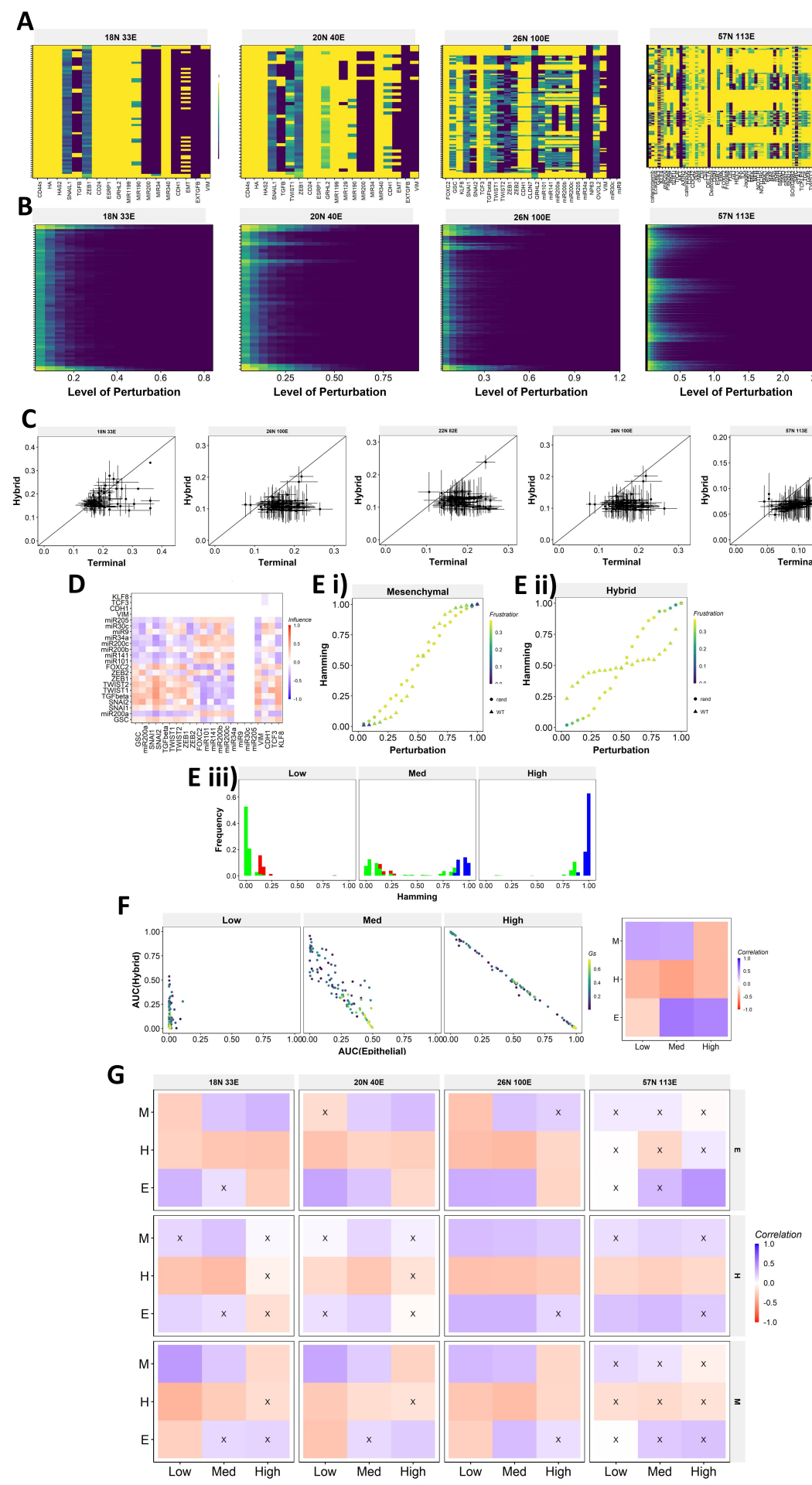
Fig S7. (A) Single node perturbation coherence heatmaps for WT EMP networks (B) Multi node perturbation coherence heatmaps for WT EMP networks (C) Half-minimum perturbation for terminal and hybrid states for the random networks. Each dot represents mean half-minimum perturbation for terminal and hybrid states for a network, and the bars represent standard deviation. (D) Influence matrix of the random network with low group strength taken as the representative case for Fig 5E (E) Representative cases of comparison of transition dynamics for Mesenchymal and hybrid steady states between WT and random network. (F) Same as Fig 6F but for Mesenchymal phenotype as the beginning. (G) Heatmaps depicting the correlation between mean group strength and AUC for combinations of perturbation strengths and final phenotypes for all networks. 